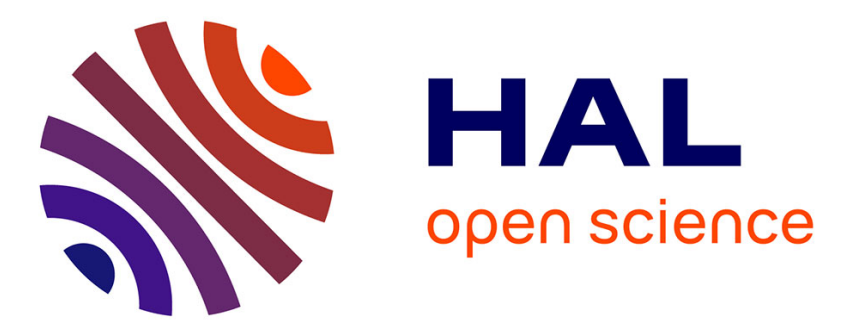

\title{
Apprehending the effects of mechanical deformations in cardiac electrophysiology: A homogenization approach
}

Annabelle Collin, Sébastien Imperiale, Philippe Moireau, Jean-Frédéric

Gerbeau, Dominique Chapelle

\section{- To cite this version:}

Annabelle Collin, Sébastien Imperiale, Philippe Moireau, Jean-Frédéric Gerbeau, Dominique Chapelle. Apprehending the effects of mechanical deformations in cardiac electrophysiology: A homogenization approach. Mathematical Models and Methods in Applied Sciences, 2019, 29 (13), pp.2377-2417. 10.1142/S0218202519500490 . hal-02267941v2

\section{HAL Id: hal-02267941 \\ https://hal.inria.fr/hal-02267941v2}

Submitted on 14 Dec 2019

HAL is a multi-disciplinary open access archive for the deposit and dissemination of scientific research documents, whether they are published or not. The documents may come from teaching and research institutions in France or abroad, or from public or private research centers.
L'archive ouverte pluridisciplinaire HAL, est destinée au dépôt et à la diffusion de documents scientifiques de niveau recherche, publiés ou non, émanant des établissements d'enseignement et de recherche français ou étrangers, des laboratoires publics ou privés. 


\title{
Apprehending the effects of mechanical deformations in cardiac electrophysiology - An homogenization approach
}

\author{
Annabelle Collin ${ }^{c, a}$, Sébastien Imperiale ${ }^{a, b}$, Philippe Moireau $^{a, b}$, Jean-Frédéric Gerbeau ${ }^{a, d}$ \\ and Dominique Chapelle ${ }^{a, b}$ \\ ${ }^{a}$ Inria \\ ${ }^{b}$ LMS, CNRS, Ecole Polytechnique, Institut Polytechnique de Paris \\ ${ }^{c}$ Université de Bordeaux \\ ${ }^{d}$ Sorbonne Université, UMR 7598 LJLL
}

Published in M3AS, 29(13):2377-2417, 2019

\begin{abstract}
We follow a formal homogenization approach to investigate the effects of mechanical deformations in electrophysiology models relying on a bidomain description of ionic motion at the microscopic level. To that purpose, we extend these microscopic equations to take into account the mechanical deformations, and proceed by recasting the problem in the framework of classical two-scale homogenization in periodic media, and identifying the equations satisfied by the first coefficients in the formal expansions. The homogenized equations reveal some interesting effects related to the microstructure - and associated with a specific cell problem to be solved to obtain the macroscopic conductivity tensors - in which mechanical deformations play a non-trivial role, i.e. do not simply lead to a standard bidomain problem posed in the deformed configuration. We then present detailed numerical illustrations of the homogenized model with coupled cardiac electrical-mechanical simulations - all the way to ECG simulations - albeit without taking into account the abundantly-investigated effect of mechanical deformations in ionic models, in order to focus here on other effects. And in fact our numerical results indicate that these other effects are numerically of a comparable order, and therefore cannot be disregarded.
\end{abstract}

\section{Introduction}

Cardiac electrophysiology modeling is a very mature field of research, and the so-called bidomain model - introduced in the late 1970's, see [73] - is nowadays considered as a reference model, while some approximations thereof are often considered, with the so-called monodomain model $[17,65]$, in particular, and also some specific forms of eikonal equations [32, 33, 37]. Concurrently, cardiac mechanical modeling has made tremendous advances over the past decades, and complete modeling frameworks are now available to represent the detailed mechanical phenomena induced by electrophysiology over entire heartbeats, including for predictive purposes in clinical applications where pathological behavior must be incorporated in the model, see [12] and references therein. However, although the deformation of cardiac tissue is well-known to be very substantial and while computer power is no longer an obstacle for considering fully coupled multiphysics problems, the question of the full coupling between cardiac electrophysiology and mechanics is still partly open. More specifically, whereas the mechanisms by which electrophysiology entails tissue contraction and relaxation have been actively investigated, the reverse effects by which mechanics impacts electrophysiology - i.e. the so-called "mechano-electrical 
feedback" - remain to be fully elucidated. More precisely, the effect of mechanics on ionic models - i.e. ordinary differential equations aimed at describing the local exchanges of ions between various cellular compartments, including through the cell membranes - has received significant attention, see [34, 48], but by contrast the effects on conservation laws (of charges and ions) that govern the spatial variations of all quantities of interest have not been as actively studied. We refer to [16] for a detailed survey on the latter aspect, see also [14, 15, 21, 61].

Clearly, a major difficulty for elucidating the effects of mechanical deformations on the bidomain model, in particular, is that the conservation laws - pertaining to ions and associated electrical charges - incorporated in this model are in fact posed at the microscopic level, and are not directly visible in the macroscopic bidomain model equations. These macroscopic bidomain equations are, indeed, the result of a complex homogenization process starting from a microscopic level associated with the cellular scale at which intra- and extracellular domains - separated by the cell membrane - can be geometrically defined [18, 49,63]. Furthermore, the derivation of the macroscopic bidomain equations via this homogenization process has been performed under various assumptions, among which the absence of mechanical deformations. There lies the fundamental motivation and program of the present paper, i.e. it is essential to go back to the origins of the bidomain model to ultimately elucidate the effects of mechanical deformations on this model. In so doing, we will also revisit the other underlying assumptions of the bidomain model, and in particular the assumption according to which ionic diffusion can be neglected compared to drift induced by the electrical fields in order to eliminate ionic concentrations from the final partial differential equations (PDEs), except in the ionic models, of course. We will note in passing that this assumption is not straightforward to justify, although this is not the subject of this paper. We emphasize, indeed, that it is not our objective in this work to question - and a fortiori to justify - the underlying assumptions of the microscopic bidomain model, nor the asymptotic assumptions laying the ground for the homogenization, in particular the leading orders sought in the various terms. Our aim is to focus on the effects of mechanical deformations in this homogenization procedure, and for completeness we only allow ourselves to consider the variant of equations obtained without neglecting ionic diffusion. We will therefore proceed to a formal homogenization derivation when including mechanical deformations and without neglecting ionic diffusion, and finally obtain a complete system of macroscopic equations in which the conductivity tensors are given by a cell problem - i.e. posed at the microscopic level - in which the solution depends on the mechanical deformation. In the final result, we can then infer a simpler model by neglecting ionic diffusion, which again allows to eliminate ionic concentrations from the PDEs, albeit this transfers the cell-dependent tensor to the new macroscopic bidomain equations.

The resulting homogenized model can then be compared to previously-proposed formulations, and in particular to that obtained when directly posing the usual macroscopic bidomain equations in the deformed configuration, as considered and numerically investigated in [16]. We will, indeed, find two major differences with such "macroscopic-only" formulations: (1) there should be no convection term in the Lagrangian form of the bidomain equations, or in other words the membrane equations are intrinsically Lagrangian, and (2) the standard transformation formula for Lagrangian vs. Eulerian conductivity tensors does not hold. Moreover, for the second difference the homogenized model can be used to assess the error made when using the standard transformation formula.

The outline of the paper is as follows. In Section 2, we recall the microscopic model that underlies the bidomain model, and we extend these equations to take into account the mechanical deformations. Then, in Section 3, we recast the problem in the framework of classical two-scale homogenization in periodic media, and identify the equations satisfied by the first coefficients in the formal expansions. Finally, in Section 4, we present detailed numerical illustrations of 
the homogenized model with coupled cardiac electrical-mechanical simulations, all the way to ECG simulations, before giving some concluding remarks in Section 5.

\section{Microscopic model}

The purpose of this section is to recall the microscopic model - governing ionic motion and electric potentials - that underlies the bidomain model, and to derive extensions of these equations in order to take into account mechanical deformations.

\subsection{Definitions and notation}

The geometric domain occupied by the cardiac tissue at any time $t$ is denoted by $\Omega(t)$, with boundary $\partial \Omega(t)$. We use a Lagrangian description, for which we introduce a reference configuration $\Omega_{0}$ - not necessarily corresponding to $\Omega(0)$ - and the one-to-one deformation mapping $\phi$ from the reference configuration to the current configuration, giving the position of each material point over time,

$$
\underline{\phi}:\left\{\begin{array}{ccc}
\bar{\Omega}_{0} \times[0, T] & \rightarrow & \mathbb{R}^{3} \\
(\underline{\xi}, t) & \mapsto & \underline{x}(t)=\underline{\phi}(\underline{\xi}, t) .
\end{array}\right.
$$

We define the displacement $\underline{y}$ by

$$
\underline{y}(\underline{\xi}, t)=\underline{x}-\underline{\xi}=\underline{\phi}(\underline{\xi}, t)-\underline{\xi},
$$

and the deformation gradient by

$$
\underline{\underline{F}}(\underline{\xi}, t)=\underline{\underline{\nabla}} \underline{\underline{\phi}}=\underline{\underline{1}}+\underline{\underline{\nabla}}_{\underline{\xi}} \underline{y} .
$$

We will assume that $J=\operatorname{det} \underline{\underline{F}}$ is strictly positive for any $\underline{\xi} \in \bar{\Omega}_{0}$, meaning that the deformation mapping does not degenerate anywhere. Of course, this implies that $\underline{\underline{F}}$ is always invertible, in particular.

In fact, this geometric domain $\Omega_{0}$ is decomposed into two parts, i.e. $\Omega_{0}^{(i)}$ the intracellular region and $\Omega_{0}^{(e)}$ the extracellular region, see Figure 1, with the associated regions $\Omega^{(i)}(t)$ and $\Omega^{(e)}(t)$ in the deformed configuration. Geometrically, $\Omega_{0}^{(i)}$ and $\Omega_{0}^{(e)}$ are two connected domains verifying

$$
\bar{\Omega}_{0}=\bar{\Omega}_{0}^{(e)} \cup \bar{\Omega}_{0}^{(i)} \text { and } \Omega_{0}^{(e)} \cap \Omega_{0}^{(i)}=\varnothing .
$$

We suppose that the membrane separating the two regions $\Gamma_{0}=\partial \Omega_{0}^{(e)} \cap \partial \Omega_{0}^{(i)}$ is regular, and we define $\underline{n}_{0}^{(i)}$ and $\underline{n}_{0}^{(e)}$ as the unit outward-pointing normal vectors associated with $\Omega_{0}^{(e)}$ and $\Omega_{0}^{(i)}$, respectively, with of course $\underline{n}_{0}^{(i)}=-\underline{n}_{0}^{(e)}$. Accordingly, $\underline{n}^{(e)}(t)$ and $\underline{n}^{(i)}(t)$ denote the unit normal vectors on the deformed membrane $\Gamma(t)$. In what follows, for the sake of compactness we will often drop the explicit time dependence in the notation pertaining to the deformed configuration, as in $\Omega^{(e)}$ and $\Omega^{(i)}$.

As usual, the deformation mapping $\phi$ allows to transport to the deformed domain $\Omega(t)$ any field $f(\underline{\xi}, t)$ defined over the reference domain $\Omega_{0}$, by simply considering $f\left(\underline{\phi}^{-1}(\underline{x}), t\right)$. And conversely of course, any field primarily defined over $\Omega(t)$ can be transported back to $\Omega_{0}$ by composition with $\phi$ itself. For the sake of compactness, we will use the same notation $f$ to indifferently refer to either field considered in $\Omega_{0}$ or $\Omega(t)$. Nevertheless, some special care is required in this context as regards time partial derivatives, and therefore we will use the notation 


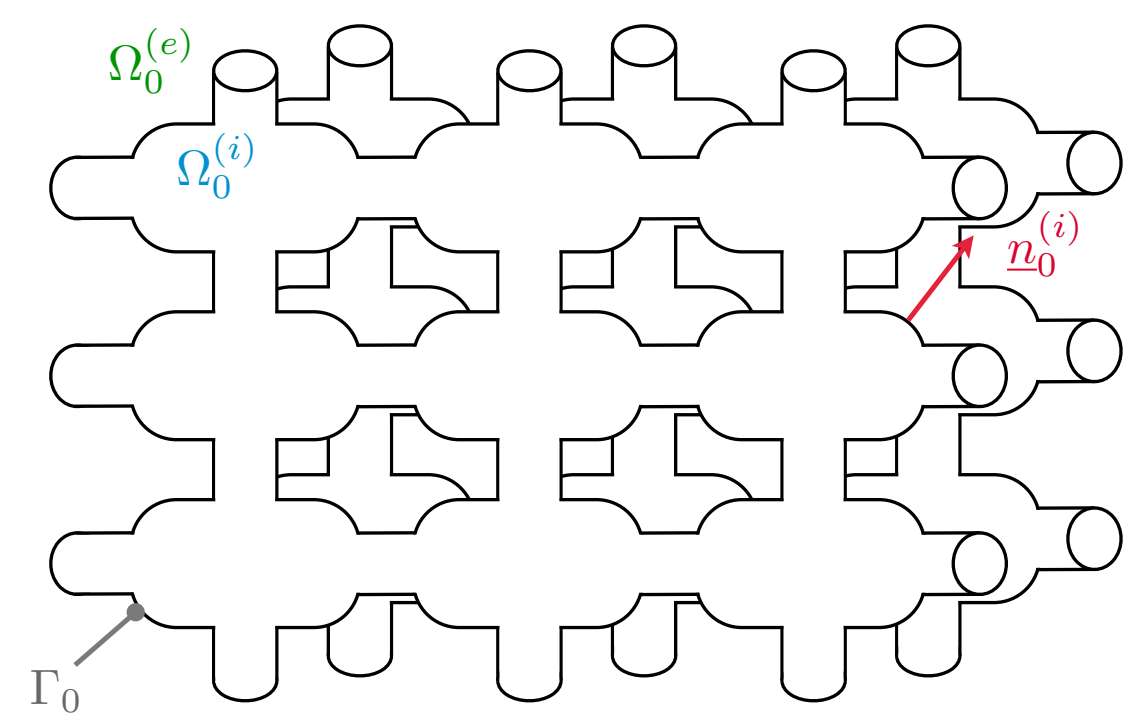

Figure 1: Intracellular and extracellular regions of the heart.

$\left.\frac{\partial f}{\partial t}\right|_{\underline{\xi}}$ or $\left.\frac{\partial f}{\partial t}\right|_{\underline{x}}$ to indicate that we are time-differentiating the field considered as a function of $(\underline{\xi}, \bar{t})$ or $(\underline{x}, \bar{t})$, respectively, with the classical transformation formula

$$
\left.\frac{\partial f}{\partial t}\right|_{\underline{\xi}}=\left.\frac{\partial f}{\partial t}\right|_{\underline{x}}+\underline{v}_{s} \cdot \underline{\nabla}_{\underline{x}} f
$$

denoting $\underline{v}_{s}=\left.\frac{\partial \underline{y}}{\partial t}\right|_{\underline{\xi}}$ the material velocity. We will also use the terminology of Lagrangian versus Eulerian derivatives to refer to derivatives of fields considered as functions of $(\underline{\xi}, t)$ or $(\underline{x}, t)$, respectively.

\subsection{General model of ion motion}

In this section, we give an overview of the derivation of the equations governing ionic motion in the intracellular and extracellular regions, namely, the so-called Nernst-Planck-Poisson equations. For more details we refer e.g. to [36].

We consider ion motion in an isothermal medium with negligible solvent flows. We suppose that we have $N^{(i)}$ and $N^{(e)}$ different species of mobile ions in the intra- and extracellular regions, respectively. The individual conservation gives, in each region,

$$
\left.\frac{\partial c_{k}^{(\alpha)}}{\partial t}\right|_{\underline{x}}+\underline{\nabla}_{\underline{x}} \cdot \underline{\jmath}_{k}^{(\alpha)}=0, k=1, \ldots, N^{(\alpha)}, \alpha=i, e,
$$

where $c_{k}^{(\alpha)}, \underline{\jmath}_{k}^{(\alpha)}=c_{k}^{(\alpha)} \underline{v}_{k}^{(\alpha)}$ are the concentration and the flux of the $k$-th ionic species, respectively, in the given region, and with $\underline{v}_{k}^{(\alpha)}$ the corresponding velocity. Denoting by $\underline{v}_{k, r}^{(\alpha)}$ the relative velocity of the ions with respect to the substrate, we will use the decompositions

$$
\underline{v}_{k}^{(\alpha)}=\underline{v}_{s}+\underline{v}_{k, r}^{(\alpha)}, \quad \underline{\jmath}_{k}^{(\alpha)}=\underline{\jmath}_{k, s}^{(\alpha)}+\underline{\jmath}_{k, r}^{(\alpha)}=c_{k}^{(\alpha)}\left(\underline{v}_{s}+\underline{v}_{k, r}^{(\alpha)}\right) .
$$

The total charge density $\rho^{(\alpha)}=\rho_{b}^{(\alpha)}+\mathrm{F} \sum_{k} z_{k} c_{k}^{(\alpha)}$ - with $\rho_{b}^{(\alpha)}$ the background charge density (independent of the mobile ions), $\mathrm{F}$ the Faraday constant, and $z_{k}$ the signed number of 
elementary charges carried by this ion - is related to the electric field $\underline{E}^{(\alpha)}$ by the Gauss law of electrostatics

$$
\underline{\nabla}_{\underline{x}} \cdot \underline{E}^{(\alpha)}=\frac{\rho^{(\alpha)}}{\varepsilon_{0} \varepsilon_{s}}=\frac{1}{\varepsilon_{0} \varepsilon_{s}}\left(\rho_{b}^{(\alpha)}+\mathrm{F} \sum_{k=1}^{N^{(\alpha)}} z_{k} c_{k}^{(\alpha)}\right),
$$

with $\varepsilon_{s}$ the relative permittivity of the medium, and $\varepsilon_{0}$ the permittivity of free space.

In order to determine the flux $\underline{j}_{k}^{(\alpha)}=c_{k}^{(\alpha)} \underline{v}_{k}^{(\alpha)}$ of the species $k$, we apply Newton's second law to an ion of this species. The forces undergone by this ion are:

- the friction force $\underline{F}_{k}^{\text {frict }}=-\frac{\underline{v}_{k, r}^{(\alpha)}}{m_{k}}$, where $m_{k}$ denotes the so-called mobility of the ion;

- the electric force $\underline{F}_{k}^{\text {elec }}=z_{k} q \underline{E}^{(\alpha)}$, with $q$ the charge of a proton;

- the diffusion force, which reads, according to Einstein's diffusion law, $\underline{F}_{k}^{\text {dif }}=-\frac{k_{B} T}{c_{k}^{(\alpha)}} \underline{\nabla}_{\underline{x}} c_{k}^{(\alpha)}$, where $k_{B}$ denotes the Boltzmann constant and $T$ the absolute temperature.

Neglecting the inertia effect, we then have the equilibrium equation

$$
\underline{F}_{k}^{\text {dif }}+\underline{F}_{k}^{\text {frict }}+\underline{F}_{k}^{\text {elec }}=0,
$$

and we infer

$$
-\frac{k_{B} T}{c_{k}^{(\alpha)}} \underline{\nabla}_{\underline{x}} c_{k}^{(\alpha)}+z_{k} q \underline{E}^{(\alpha)}=\frac{\underline{v}_{k, r}^{(\alpha)}}{m_{k}} .
$$

Therefore, for the flux $\underline{\jmath}_{k}^{(\alpha)}=c_{k}^{(\alpha)} \underline{v}_{k}^{(\alpha)}$ of the species $k$, we obtain the so-called Nernst-Planck equation

$$
\underline{\jmath}_{k}^{(\alpha)}=-m_{k} k_{B} T \underline{\nabla}_{\underline{x}} c_{k}^{(\alpha)}+m_{k} z_{k} c_{k}^{(\alpha)} q \underline{E}^{(\alpha)}+c_{k}^{(\alpha)} \underline{v}_{s},
$$

which can be rephrased as

$$
\underline{\jmath}_{k}^{(\alpha)}=-D_{k}\left(\underline{\nabla}_{\underline{x}} c_{k}^{(\alpha)}-\frac{q z_{k} c_{k}^{(\alpha)}}{k_{B} T} \underline{E}^{(\alpha)}\right)+c_{k}^{(\alpha)} \underline{v}_{s}
$$

with $D_{k}=m_{k} k_{B} T$ the diffusion coefficient of the species $k$. The electric field is related to an electric potential $u$ by $\underline{E}^{(\alpha)}=-\underline{\nabla}_{\underline{x}} u^{(\alpha)}$. Using (3), (4) and (5), we finally obtain for $k=1, \ldots, N^{(\alpha)}$

$$
\mid \begin{aligned}
& - \text { Charge conservation } \\
& \left.\quad \frac{\partial c_{k}^{(\alpha)}}{\partial t}\right|_{\underline{x}}=-\underline{\nabla}_{\underline{x}} \cdot \underline{j}_{k}^{(\alpha)} ; \\
& - \text { Drift-diffusion flux } \\
& \quad \underline{j}_{k}^{(\alpha)}=-D_{k}\left(\underline{\nabla}_{\underline{x}} c_{k}^{(\alpha)}+\frac{q z_{k} c_{k}^{(\alpha)}}{k_{B} T} \underline{\nabla}_{\underline{x}} u^{(\alpha)}\right)+c_{k}^{(\alpha)} \underline{v}_{s} \\
& - \text { Gauss law } \\
& \quad \Delta_{\underline{x}} u^{(\alpha)}=-\frac{1}{\varepsilon_{0} \varepsilon_{s}}\left(\rho_{b}^{(\alpha)}+\mathrm{F} \sum_{k=1}^{N^{(\alpha)}} z_{k} c_{k}^{(\alpha)}\right) .
\end{aligned}
$$

This system of equations is a straightforward extension of the so-called Nernst-Planck-Poisson system, with here the additional term $c_{k}^{(\alpha)} \underline{v}_{s}$ resulting from the substrate motion. 


\section{$2.3 \quad$ Electroneutrality}

By examining the orders of magnitude of the physical parameters in the above Gauss law, compared to the time and space scales in consideration, it can be argued - and justified by a mathematical asymptotic analysis, indeed $[43,62]$ - that electroneutrality must prevail away from the membranes, i.e.

$$
\rho_{b}^{(\alpha)}+\mathrm{F} \sum_{k=1}^{N^{(\alpha)}} z_{k} c_{k}^{(\alpha)}=0,
$$

everywhere in the interior of the intra- and extracellular regions. This equation should be considered as a substitute for the Gauss law - and not together with it, which would incorrectly imply $\Delta_{\underline{x}} u^{(\alpha)}=0$ - and therefore leaves the electric potential undetermined. In order to obtain an equation for the electric potential, we can instead consider the total electric current $\underline{\jmath}^{(\alpha)}=\mathrm{F} \sum_{k=1}^{N^{(\alpha)}} z_{k} \underline{\jmath}_{k}^{(\alpha)}$. Summing each of the first two equations of (6) over the ionic species with the same weights $\mathrm{F} z_{k}$, and using the electroneutrality, we obtain

$$
\underline{\jmath}^{(\alpha)}=-\left(\sigma^{(\alpha)} \underline{\nabla}_{\underline{x}} u^{(\alpha)}+\mathrm{F} \sum_{k=1}^{N^{(\alpha)}} z_{k} D_{k} \underline{\nabla}_{\underline{x}} c_{k}^{(\alpha)}+\rho_{b}^{(\alpha)} \underline{v}_{s}\right),
$$

with $\sigma^{(\alpha)}=\mathrm{F} q \sum_{k=1}^{N^{(\alpha)}} m_{k}\left(z_{k}\right)^{2} c_{k}^{(\alpha)}$ the total effective conductivity, and also

$$
\underline{\nabla}_{\underline{x}} \cdot \underline{\jmath}^{(\alpha)}=\left.\frac{\partial \rho_{b}^{(\alpha)}}{\partial t}\right|_{\underline{x}}
$$

Alternatively, decomposing the electric current into $\underline{\jmath}^{(\alpha)}=\underline{\jmath}_{s}^{(\alpha)}+\underline{\jmath}_{r}^{(\alpha)}$ with

$$
\underline{\jmath}_{s}^{(\alpha)}=\mathrm{F}\left(\sum_{k=1}^{N^{(\alpha)}} z_{k} c_{k}^{(\alpha)}\right) \underline{v}_{s}=-\rho_{b}^{(\alpha)} \underline{v}_{s},
$$

and using the conservation of the background charge density carried by the substrate, i.e.

$$
\left.\frac{\partial \rho_{b}^{(\alpha)}}{\partial t}\right|_{\underline{x}}+\underline{\nabla}_{\underline{x}} \cdot \rho_{b}^{(\alpha)} \underline{v}_{s}=0
$$

we get

$$
\underline{\jmath}_{r}^{(\alpha)}=-\left(\sigma^{(\alpha)} \underline{\nabla}_{\underline{x}} u^{(\alpha)}+\mathrm{F} \sum_{k=1}^{N^{(\alpha)}} z_{k} D_{k} \underline{\nabla}_{\underline{x}} c_{k}^{(\alpha)}\right),
$$

and

$$
\underline{\nabla}_{\underline{x}} \cdot \underline{j}_{r}^{(\alpha)}=0 .
$$

These two equations can be seen as those governing electric potentials, instead of the Gauss law.

\subsection{Transmembrane equations}

The ions that hit the membrane can be blocked on the surface on each side, or cross the membrane through so-called ionic channels. We denote by $\mu_{k}^{(\alpha)}$ the molar density per unit surface of the reference configuration for species $k$ on either side, and by $I_{k}^{i o n}$ the molar quantity crossing the membrane from $\Omega^{(i)}$ to $\Omega^{(e)}$, per unit time and per unit surface of the reference configuration. 
Considering an arbitrary subpart of the membrane $\gamma_{0} \subset \Gamma_{0}$ in the reference configuration, associated with $\gamma(t) \subset \Gamma(t)$ in the deformed configuration, we write the conservation of species $k$ attached to the internal side of $\gamma_{0}$. Here, conservation dictates that the variation of molar quantity attached to the membrane equals the quantity crossing the membrane from $\Omega^{(e)}$ to $\Omega^{(i)}$ plus the quantity associated with ions coming from $\Omega^{(i)}$ and hitting the membrane, i.e.

$$
\frac{d}{d t} \int_{\gamma_{0}} \mu_{k}^{(i)} d \Gamma_{0}=-\int_{\gamma_{0}} I_{k}^{i o n} d \Gamma_{0}+\int_{\gamma} \underline{\jmath}_{k, r}^{(i)} \cdot \underline{n}^{(i)} d \Gamma
$$

Note that this assumes that ions attached to the membrane cannot slide on this surface, hence there is no contribution coming from the rest of the membrane through $\partial \gamma_{0}$. Changing variables in the last integral, we directly obtain

$$
\left.\int_{\gamma_{0}} \frac{\partial \mu_{k}^{(i)}}{\partial t}\right|_{\underline{\xi}} d \Gamma_{0}=-\int_{\gamma_{0}} I_{k}^{i o n} d \Gamma_{0}+\int_{\gamma_{0}} \underline{\jmath}_{k, r}^{(i)} \cdot \underline{n}^{(i)} J\left\|\underline{\underline{F}}^{-T} \cdot \underline{n}_{0}^{(i)}\right\| d \Gamma_{0},
$$

where we recall that we omit writing the composition by the deformation mapping. Since $\gamma_{0} \subset \Gamma_{0}$ is arbitrary, we now infer

$$
\left.\frac{\partial \mu_{k}^{(i)}}{\partial t}\right|_{\underline{\xi}}=-I_{k}^{i o n}+s_{\Gamma} \underline{\jmath}_{k, r}^{(i)} \cdot \underline{n}^{(i)},
$$

everywhere on $\Gamma_{0}$ (or $\Gamma$ ), where $s_{\Gamma}=J\left\|\underline{\underline{F}}^{-T} \cdot \underline{n}_{0}^{(\alpha)}\right\|$ represents the stretch ratio of the membrane in the current configuration with respect to the reference configuration. Finally, since a similar conservation can be written on the external side of the membrane, we have

$$
\left\{\begin{array}{l}
\left.\frac{\partial \mu_{k}^{(i)}}{\partial t}\right|_{\underline{\xi}}+I_{k}^{i o n}=s_{\Gamma} \underline{\jmath}_{k, r}^{(i)} \cdot \underline{n}^{(i)}, \quad \text { on } \Gamma \\
\left.\frac{\partial \mu_{k}^{(e)}}{\partial t}\right|_{\underline{\xi}}-I_{k}^{i o n}=-s_{\Gamma} \underline{g}_{k, r}^{(e)} \cdot \underline{n}^{(i)}, \quad \text { on } \Gamma
\end{array}\right.
$$

We emphasize that the time partial derivatives appearing in this system are to be understood at $\xi$ fixed, i.e. - when following a given material point of the membrane - which is intrinsically justified by the fact that ionic channels are physically attached to the membrane. Note in passing that the transformation formula (2) cannot be applied to derive an equivalent equation with a Eulerian time derivative, since the densities $\mu_{k}^{(\alpha)}$ are only defined on the membrane that is moving in the Eulerian reference frame.

Likewise, introducing

$$
I_{\text {tot }}^{i o n}=\mathrm{F} \sum_{k=1}^{N} z_{k} I_{k}^{i o n}, \quad \mu^{(\alpha)}=\mathrm{F} \sum_{k=1}^{N} z_{k} \mu_{k}^{(\alpha)},
$$

i.e. , the total flux of charge and surface charge per unit surface of the reference configuration, we can directly infer the conservation of total charge as

$$
\left\{\begin{array}{l}
\left.\frac{\partial \mu^{(i)}}{\partial t}\right|_{\underline{\xi}}+I_{t o t}^{i o n}=s_{\Gamma} \underline{\jmath}_{r}^{(i)} \cdot \underline{n}^{(i)} \\
\left.\frac{\partial \mu^{(e)}}{\partial t}\right|_{\underline{\xi}}-I_{t o t}^{i o n}=-s_{\Gamma} \underline{g}_{r}^{(e)} \cdot \underline{n}^{(i)} .
\end{array}\right.
$$


We then make two additional classical assumptions on the surface charges. Namely, any charge accumulation on one side of the membrane is automatically balanced by an opposite charge on the other side

$$
\mu^{(i)}+\mu^{(e)}=0
$$

and the membrane behaves as a capacitance, i.e. the surface charge is linearly proportional to the transmembrane potential

$$
\mu^{(i)}=C_{m} V_{m},
$$

where $V_{m}=u^{(i)}-u^{(e)}$ denotes the transmembrane potential and $C_{m}$ the capacitance per unit area of the membrane. We obtain

$$
\left\{\begin{aligned}
\left.C_{m} \frac{\partial V_{m}}{\partial t}\right|_{\underline{\xi}}+I_{t o t}^{i o n} & =s_{\Gamma} \underline{\jmath}_{r}^{(i)} \cdot \underline{n}^{(i)} \\
\underline{\jmath}_{r}^{(i)} \cdot \underline{n}^{(i)} & =\underline{\jmath}_{r}^{(e)} \cdot \underline{n}^{(i)}
\end{aligned}\right.
$$

To complete the modeling, we need some boundary conditions on the membrane for the ionic flux equations, i.e. equations relating volume-distributed ionic concentrations and the quantities characterizing the state of the membrane. It can be justified by asymptotic analysis arguments that the surface concentration of charge associated with each ionic species in the layer is in proportion to its respective adjacent volume concentration weighed by the square of the charge number [44], i.e.

$$
\frac{\mathrm{F} z_{k} \mu_{k}^{(\alpha)}}{\mu^{(\alpha)}}=\frac{\left(z_{k}\right)^{2} c_{k}^{(\alpha)}}{\sum_{\ell}\left(z_{\ell}\right)^{2} c_{\ell}^{(\alpha)}} \Longleftrightarrow \mu_{k}^{(\alpha)}=\frac{z_{k} c_{k}^{(\alpha)}}{\sum_{\ell}\left(z_{\ell}\right)^{2} c_{\ell}^{(\alpha)}} \frac{\mu^{(\alpha)}}{\mathrm{F}},
$$

where we see that - depending on the ionic charges - some surface concentrations can be negative, which amounts to a deficit of this species with respect to a reference concentration.

\subsection{Summary of equations}

Gathering the above equations, we obtain for the main unknowns $c_{k}^{(\alpha)}$ and $u^{(\alpha)}$

$$
\begin{cases}\left.\frac{\partial c_{k}^{(\alpha)}}{\partial t}\right|_{\underline{x}}=-\underline{\nabla}_{\underline{x}} \cdot \underline{\jmath}_{k}^{(\alpha)}, & \text { in } \Omega^{(\alpha)} \\ \underline{\jmath}_{k}^{(\alpha)}=-D_{k}\left(\underline{\nabla}_{\underline{x}} c_{k}^{(\alpha)}+\frac{q z_{k} c_{k}^{(\alpha)}}{k_{B} T} \underline{\nabla}_{\underline{x}} u^{(\alpha)}\right)+c_{k}^{(\alpha)} \underline{v}_{s}, & \text { in } \Omega^{(\alpha)} \\ s_{\Gamma} \underline{\jmath}_{k, r}^{(i)} \cdot \underline{n}^{(i)}=\left.\frac{\partial \mu_{k}^{(i)}}{\partial t}\right|_{\underline{\xi}}+I_{k}^{i o n}, & \text { on } \Gamma \\ s_{\Gamma} \underline{\jmath}_{k, r}^{(e)} \cdot \underline{n}^{(e)}=\left.\frac{\partial \mu_{k}^{(e)}}{\partial t}\right|_{\underline{\xi}}-I_{k}^{i o n}, & \text { on } \Gamma\end{cases}
$$

and

$$
\begin{cases}\underline{\nabla}_{\underline{x}} \cdot \underline{\jmath}_{r}^{(\alpha)}=0, & \text { in } \Omega^{(\alpha)} \\ \underline{\jmath}_{r}^{(\alpha)}=-\left(\sigma^{(\alpha)} \underline{\nabla}_{\underline{x}} u^{(\alpha)}+\mathrm{F} \sum_{k=1}^{N^{(\alpha)}} z_{k} D_{k} \underline{\nabla}_{\underline{x}} c_{k}^{(\alpha)}\right), & \text { in } \Omega^{(\alpha)} \\ s_{\Gamma} \underline{\jmath}_{r}^{(i)} \cdot \underline{n}^{(i)}=-s_{\Gamma} \underline{\jmath}_{r}^{(e)} \cdot \underline{n}^{(e)}=\left.C_{m} \frac{\partial V_{m}}{\partial t}\right|_{\underline{\xi}}+I_{\text {tot }}^{i o n}, & \text { on } \Gamma\end{cases}
$$


In addition, the auxiliary unknowns $\mu_{k}^{(\alpha)}$ and $\mu^{(\alpha)}$ - representing surface densities of ions and charges on the membrane - are given by

$$
\begin{cases}\mu_{k}^{(\alpha)}=\frac{z_{k} c_{k}^{(\alpha)}}{\sum_{\ell}\left(z_{\ell}\right)^{2} c_{\ell}^{(\alpha)}} \frac{\mu^{(\alpha)}}{\mathrm{F}}, & \text { on } \Gamma \\ \mu^{(i)}=-\mu^{(e)}=C_{m} V_{m}, & \text { on } \Gamma\end{cases}
$$

\subsection{Lagrangian form}

Alternatively, we can derive a Lagrangian form of the above equations, i.e. written entirely with respect to the space variable associated with the reference configuration. To that purpose, as is standard in the derivation of conservation laws in moving domains [69], it is classical to introduce the following modified concentration and (relative) flux quantities

$$
C_{k}^{(\alpha)}=J c_{k}^{(\alpha)}, \quad \underline{J}_{k}^{(\alpha)}=J \underline{\underline{F}}^{-1} \cdot \underline{\jmath}_{k, r}^{(\alpha)} .
$$

The conservation laws in each region then directly read

$$
\left.\frac{\partial C_{k}^{(\alpha)}}{\partial t}\right|_{\underline{\xi}}+\underline{\nabla}_{\underline{\xi}} \cdot \underline{J}_{k}^{(\alpha)}=0
$$

with the Lagrangian flux given by

$$
\underline{J}_{k}^{(\alpha)}=-J D_{k}\left(\underline{\underline{F}}^{T} \cdot \underline{\underline{F}}\right)^{-1} \cdot\left(\underline{\nabla}_{\underline{\xi}}\left(J^{-1} C_{k}^{(\alpha)}\right)+\frac{q z_{k}\left(J^{-1} C_{k}^{(\alpha)}\right)}{k_{B} T} \underline{\nabla}_{\underline{\xi}} u^{(\alpha)}\right) .
$$

For the boundary conditions, recalling that $\underline{\jmath}_{k, r}^{(\alpha)} \cdot \underline{n}^{(\alpha)} d \Gamma=\underline{J}_{k}^{(\alpha)} \cdot \underline{n}_{0}^{(\alpha)} d \Gamma_{0}$ and $d \Gamma=s_{\Gamma} d \Gamma_{0}$, we infer

$$
\left\{\begin{array}{l}
\underline{J}_{k}^{(i)} \cdot \underline{n}_{0}^{(i)}=\left.\frac{\partial \mu_{k}^{(i)}}{\partial t}\right|_{\underline{\xi}}+I_{k}^{i o n} \\
\underline{J}_{k}^{(e)} \cdot \underline{n}_{0}^{(e)}=\left.\frac{\partial \mu_{k}^{(e)}}{\partial t}\right|_{\underline{\xi}}-I_{k}^{i o n} .
\end{array}\right.
$$

Likewise, the equations governing the electric currents read

$$
\left\{\begin{array}{l}
\underline{\nabla}_{\underline{\xi}} \cdot \underline{J}^{(\alpha)}=0 \\
\underline{J}^{(\alpha)}=-J\left(\underline{\underline{F}}^{T} \cdot \underline{\underline{F}}\right)^{-1} \cdot\left(\sigma^{(\alpha)} \underline{\nabla}_{\underline{\xi}} u^{(\alpha)}+\mathrm{F} \sum_{k=1}^{N^{(\alpha)}} z_{k} D_{k} \underline{\nabla}_{\underline{\xi}}\left(J^{-1} C_{k}^{(\alpha)}\right)\right), \\
\underline{J}^{(i)} \cdot \underline{n}_{0}^{(i)}=-\underline{J}^{(e)} \cdot \underline{n}_{0}^{(e)}=\left.C_{m} \frac{\partial V_{m}}{\partial t}\right|_{\underline{\xi}}+I_{t o t}^{i o n} .
\end{array}\right.
$$

\subsection{Bidomain model simplifications}

In order to derive the classical bidomain equations, it is assumed that diffusion can be neglected compared to drift in ionic fluxes [43]. Whether or not this assumption is valid in practice is an open question that goes beyond the scope of this paper. Nevertheless, under this 
assumption System (19) directly yields

$$
\begin{cases}\underline{\nabla}_{\underline{x}} \cdot\left(\sigma^{(\alpha)} \underline{\nabla}_{\underline{x}} u^{(\alpha)}\right)=0, & \text { in } \Omega^{(\alpha)} \\ \sigma^{(i)} \underline{\nabla}_{\underline{x}} u^{(i)} \cdot \underline{n}^{(i)}=\sigma^{(e)} \underline{\nabla}_{\underline{x}} u^{(e)} \cdot \underline{n}^{(i)}, & \text { on } \Gamma \\ -s_{\Gamma} \sigma^{(i)} \underline{\nabla}_{\underline{x}} u^{(i)} \cdot \underline{n}^{(i)}=\left.C_{m} \frac{\partial V_{m}}{\partial t}\right|_{\underline{\xi}}+I_{t o t}^{i o n}, & \text { on } \Gamma\end{cases}
$$

Alternatively, we have the following Lagrangian form

$$
\begin{cases}\underline{\nabla}_{\underline{\xi}} \cdot\left(J \sigma^{(\alpha)}\left(\underline{\underline{F}}^{T} \cdot \underline{\underline{F}}\right)^{-1} \cdot \underline{\nabla}_{\underline{\xi}} u^{(\alpha)}\right)=0, & \text { in } \Omega_{0}^{(\alpha)} \\ \sigma^{(i)}\left(\left(\underline{\underline{F}}^{T} \cdot \underline{\underline{F}}\right)^{-1} \cdot \underline{\nabla}_{\underline{\xi}} u^{(i)}\right) \cdot \underline{n}_{0}^{(i)}=\sigma^{(e)}\left(\left(\underline{\underline{F}}^{T} \cdot \underline{\underline{F}}\right)^{-1} \cdot \underline{\nabla}_{\underline{\xi}} u^{(e)}\right) \cdot \underline{n}_{0}^{(i)}, & \text { on } \Gamma_{0} \\ -J \sigma^{(i)}\left(\left(\underline{\underline{F}}^{T} \cdot \underline{\underline{F}}\right)^{-1} \cdot \underline{\nabla}_{\underline{\xi}} u^{(i)}\right) \cdot \underline{n}_{0}^{(i)}=\left.C_{m} \frac{\partial V_{m}}{\partial t}\right|_{\underline{\xi}}+I_{t o t}^{i o n}, & \text { on } \Gamma_{0}\end{cases}
$$

We recall that the effective conductivities $\sigma^{(\alpha)}$ are defined by

$$
\sigma^{(\alpha)}=\mathrm{F} q \sum_{k=1}^{N^{(\alpha)}} m_{k}\left(z_{k}\right)^{2} c_{k}^{(\alpha)},
$$

which implies that these conductivities are not constant in general, because ionic concentrations are not, both space- and time-wise. Nevertheless, the assumption of constant conductivities made in the bidomain model is easier to justify than the negligible effect of diffusion in ionic fluxes, because the latter involves concentration gradients over very small (cellular) length scales.

We further point out that the only difference between (25) and the classical (microscopic) bidomain equations in a fixed domain lies in the fact that the time partial derivative of the transmembrane potential is here taken at fixed $\underline{\xi}$, whereas the space derivatives are considered with respect to $\underline{x}$, the position in the deformed domain.

\subsection{Ionic models and mechanical effects}

So far, we have not dwelled on the description of the transmembrane currents $I_{t o t}^{i o n}$ and ionic fluxes $I_{k}^{i o n}$, although to date the effects of mechanical deformations on electrophysiology have mainly been considered via these terms in the literature. As our main objective in this paper is to identify and study other effects, we will here simply provide a very brief overview of these so-called ionic models.

Physiological vs. phenomenological ionic models Physiological ionic models aim at describing ionic exchanges up to some varying degree of detail. Historically, the first such model was proposed for neurons by Hodgkin and Huxley [29]. Some models were later introduced to more specifically address cardiac electrophysiology with - to cite just a few classical ones - the models proposed in $[20,50]$ for atrial cells, and the ventricle model proposed in [70] - further extended in [71] to include an improved intracellular calcium dynamics - and the model proposed in [51] that probably represents the most comprehensive human ventricle cell model published so far.

By contrast, so-called phenomenological ionic models are intended to focus on reproducing the variations of the transmembrane potential with much fewer state variables. The first 
phenomenological model was independently proposed by FitzHugh and Nagumo [22, 46] for neurons, and was later adapted to cardiac cells in [2, 64]. A more recent phenomenological model was proposed in [42], and used for ECG modeling and simulations in [6, 7]. To conclude, we mention the Minimal model for human Ventricular action potentials (MV) model that was calibrated to the mid-myocardial, epicardial and endocardial cells of the ventricular tissue. It is associated with three ionic currents, three gate variables, and governed by 28 parameters, see a further description in Section 4.2.1.

Stretch-Activated Channels In a first approximation, cardiac electrophysiology and mechanics are often assumed to be "weakly coupled", i.e. electrophysiology is taken as an input that drives the active part in the mechanical behavior. However, it is known that mechanical deformation modifies the electrical properties. This is the so-called mechano-electrical feedback (MEF), which is believed to be at work in arrhythmias and other pathologies, see e.g. [4] and references therein. One of the mechanisms of the MEF is provided by the stretch-activated channels (SAC). To model this effect, some parts of the ionic models are made dependent on the deformation, the effects of which usually being to reduce the action potential duration and amplitude, and to increase the resting transmembrane potential $[1,27]$. It is shown in [52] that the strain of the myocardium can induce automatic pacemaking activity. In [1], it is observed that the most important consequence of $\mathrm{SAC}$ is an increase in the intracellular concentration of $\mathrm{Ca}^{2+}$, and consequently an increase in contractility. This observation is supported by many studies, see the references in [1].

To close this section, we would like to underline, as in [75], that the role and mechanisms of stretch-activated channels are still not fully elucidated, and experimental findings indicate rather strong uncertainties in their characterized quantities, notwithstanding inter-species variability.

In what follows, as already mentioned we consider the ionic model as given and we disregard its direct dependence on mechanical deformations to focus on other effects and assess them.

\section{Macroscopic model}

Our objective in this section is to use an homogenization approach - based on a local periodicity assumption for cardiac cells - in order to obtain macroscopic equations for the electrical and ionic quantities, i.e. equations posed on the whole domain, instead of the above microscopic equations posed separately in the intra- and extracellular regions. In so doing, we follow the path originally proposed in [18] - see also [19] for an approach using 2-scale convergence - to derive the macroscopic bidomain equations, albeit here with two substantial extensions, i.e. : (1) for the complete equations inferred from the Nernst-Planck-Poisson system without resorting to the bidomain simplifications a priori; (2) taking into account the mechanical deformations.

\subsection{Formal homogenization in Lagrangian coordinates}

\subsubsection{Setting definition and homogenization Ansatz}

We define $Y$ as a domain that represents an idealized micro-structure corresponding to a cardiac cell. Each cell is decomposed into its intracellular part $Y^{(i)}$ and its extracellular part $Y^{(e)}$ that are separated by $\Gamma_{Y}$, with outward-pointing unit vectors $\underline{n}_{Y}^{(i)}$ and $\underline{n}_{Y}^{(e)}$, respectively. We assume that the reference domain $\bar{\Omega}_{0}=\bar{\Omega}_{0}^{(e)} \cup \bar{\Omega}_{0}^{(i)}$ and the boundary $\Gamma_{0}$ are given by a periodic repetition of entire cells - with the period parameter denoted by $\varepsilon$ and related to the 
size of the cell - namely,

$$
\bar{\Omega}_{0}^{(\alpha)}=\bigcup_{\underline{t}} \overline{\left(\varepsilon Y^{(\alpha)}+\varepsilon \underline{t}\right)} \quad \text { and } \quad \Gamma_{0}=\bigcup_{\underline{t}}\left(\varepsilon \Gamma_{Y}+\varepsilon \underline{t}\right),
$$

where $\underline{t}$ is the vector corresponding to the translation from a reference cell to each cell of the domain. The periodicity assumption is not exactly adapted to the global fiber structure of the heart, because the fiber direction varies spatially. However, we can argue that periodicity holds "locally" in the sense of [58], and we will derive the corresponding straightforward extension in the next section, while starting here with exact periodicity.

Our homogenization Ansatz is then that each main unknown of the microscopic electrophysiology problem defined on the reference domain is the sum of

- a leading term, defined everywhere in $\Omega_{0}$ and independent of $\varepsilon$;

- correction terms, also defined everywhere in $\Omega_{0}$, but dependent on $\varepsilon$ and with local variations inside each cell.

More precisely, we assume that

$$
C_{k}^{(\alpha)}(\underline{\xi}, t)=\left.C_{k, 0}^{(\alpha)}(\underline{\xi}, t)\right|_{\Omega^{(\alpha)}}+\left.\varepsilon C_{k, 1}^{(\alpha)}(\underline{\xi}, \underline{\xi} / \varepsilon, t)\right|_{\Omega^{(\alpha)}}+\left.\varepsilon^{2} C_{k, 2}^{(\alpha)}(\underline{\xi}, \underline{\xi} / \varepsilon, t)\right|_{\Omega^{(\alpha)}}+o\left(\varepsilon^{2}\right),
$$

and

$$
u^{(\alpha)}(\underline{\xi}, t)=\left.u_{0}^{(\alpha)}(\underline{\xi}, t)\right|_{\Omega^{(\alpha)}}+\left.\varepsilon u_{1}^{(\alpha)}(\underline{\xi}, \underline{\xi} / \varepsilon, t)\right|_{\Omega^{(\alpha)}}+\left.\varepsilon^{2} u_{2}^{(\alpha)}(\underline{\xi}, \underline{\xi} / \varepsilon, t)\right|_{\Omega^{(\alpha)}}+o\left(\varepsilon^{2}\right),
$$

with the correction terms $C_{k, 1}^{(\alpha)}(\underline{\xi}, \underline{\eta}, t), C_{k, 2}^{(\alpha)}(\underline{\xi}, \underline{\eta}, t), u_{1}^{(\alpha)}(\underline{\xi}, \underline{\eta}, t)$ and $u_{2}^{(\alpha)}(\underline{\xi}, \underline{\eta}, t)$ periodic in the the variable $\eta \in Y$. Note that, due to the assumption that the domains $\Omega_{0}^{(i)}$ and $\Omega_{0}^{(e)}$ are connected sets, the periodic boundary condition holds in the cell over a subpart of the boundary of non-zero measure. To simplify our study, we also assume that the deformation mapping $\underline{\phi}$ is such that its gradient $\underline{\underline{F}}$ has a leading term independent of the microstructure, which means

$$
\underline{\underline{F}}=\underline{\underline{F}} 0 \underline{\underline{\xi}}, t)+o(1) \text {. }
$$

As only the leading order term in $\underline{\underline{F}}$ will be needed in our analysis, we will not distinguish between $\underline{\underline{F}}$ and $\underline{\underline{F}}=0$, and in fact only use the notation $\underline{\underline{F}}$.

Regarding the membrane equations, we consider asymptotic assumptions consistent with those made in the homogenization of the classical bidomain equations - see [49] and [19] starting with the assumption that the capacitance parameter $C_{m}$ scales like $\varepsilon$, i.e.

$$
C_{m}=\varepsilon \widehat{C}_{m} .
$$

Therefore, with $\mu^{(i)}=-\mu^{(e)}=C_{m}\left(u^{(i)}-u^{(e)}\right)$, this justifies seeking the surface charges in the form

$$
\mu^{(\alpha)}(\underline{\xi}, t)=\left.\varepsilon\left(\mu_{0}^{(\alpha)}(\underline{\xi}, t)\right)\right|_{\Gamma_{0}}+o(\varepsilon),
$$

with the direct identification

$$
\mu_{0}^{(i)}(\underline{\xi}, t)=-\mu_{0}^{(e)}(\underline{\xi}, t)=\widehat{C}_{m} V_{m, 0}(\underline{\xi}, t),
$$

where we have introduced the notation $V_{m, 0}=u_{0}^{(i)}-u_{0}^{(e)}$ for the leading term in the expansion of the transmembrane potential $V_{m}$. Furthermore, recalling

$$
\mu_{k}^{(\alpha)}=\frac{z_{k} C_{k}^{(\alpha)}}{\sum_{\ell}\left(z_{\ell}\right)^{2} C_{\ell}^{(\alpha)}} \frac{\mu^{(\alpha)}}{\mathrm{F}},
$$


we infer similar expansions for the individual ionic surface charges, i.e.

$$
\mu_{k}^{(\alpha)}(\underline{\xi}, t)=\left.\varepsilon\left(\mu_{k, 0}^{(\alpha)}(\underline{\xi}, t)\right)\right|_{\Gamma_{0}}+o(\varepsilon)
$$

with $\mu_{k, 0}^{(\alpha)}$ also independent of $\underline{\eta}$ and given by

$$
\mu_{k, 0}^{(\alpha)}=\frac{z_{k} C_{k, 0}^{(\alpha)}}{\sum_{\ell}\left(z_{\ell}\right)^{2} C_{\ell, 0}^{(\alpha)}} \frac{\mu_{0}^{(\alpha)}}{\mathrm{F}} .
$$

Finally, in view of the membrane equations (23) it is natural to make a similar asymptotic assumption on the ionic terms $I_{k}^{i o n}$, viz.

$$
I_{k}^{i o n}(\underline{\xi}, t)=\left.\varepsilon\left(I_{k, 0}^{i o n}(\underline{\xi}, t)\right)\right|_{\Gamma_{0}}+o(\varepsilon) .
$$

We will now incorporate the equations of our homogenization Ansatz into the governing equations of Section 2.6, our major objective being to identify the equations satisfied by the leading order terms $C_{k, 0}^{(\alpha)}$ and $u_{0}^{(\alpha)}$. It should be noted, however, that these terms will be mixed with higher order terms in the equations that we will directly infer, due to space differentiation with the coexistence of the $\underline{\xi}$ and $\underline{\xi} / \varepsilon$ variables in various terms. Indeed, for any quantity $f=f_{0}(\underline{\xi}, \underline{\xi} / \varepsilon)$ with $f_{0}(\underline{\xi}, \underline{\eta})$ periodic in $\underline{\eta}$, we have

$$
\underline{\nabla}_{\underline{\xi}} f(\underline{\xi})=\underline{\nabla}_{\underline{\xi}} f_{0}(\underline{\xi}, \underline{\xi} / \varepsilon)+\varepsilon^{-1} \underline{\nabla}_{\underline{\eta}} f_{0}(\underline{\xi}, \underline{\xi} / \varepsilon) \text {. }
$$

\subsubsection{Ionic flux leading order}

Using the identity (35), we derive an expansion of the ionic fluxes (22) as follows

$$
\underline{J}_{k}^{(\alpha)}=\underline{J}_{k, 0}^{(\alpha)}+\varepsilon \underline{J}_{k, 1}^{(\alpha)}+o(\varepsilon),
$$

where

$$
\underline{J}_{k, 0}^{(\alpha)}=-J D_{k}\left(\underline{\underline{F}}^{T} \cdot \underline{\underline{F}}\right)^{-1} \cdot\left(\underline{\nabla}_{\underline{\xi}}\left(J^{-1} C_{k, 0}^{(\alpha)}\right)+J^{-1} \underline{\nabla}_{\underline{\eta}} C_{k, 1}^{(\alpha)}+\frac{q z_{k} J^{-1} C_{k, 0}^{(\alpha)}}{k_{B} T}\left(\underline{\nabla}_{\underline{\xi}} u_{0}^{(\alpha)}+\underline{\nabla}_{\underline{\eta}} u_{1}^{(\alpha)}\right)\right),
$$

and where the explicit expression of $\underline{J}_{k, 1}^{(\alpha)}$ that depends on $\left(C_{k, 0}^{(\alpha)}, C_{k, 1}^{(\alpha)}, C_{k, 2}^{(\alpha)}\right)$ and $\left(u_{0}^{(\alpha)}, u_{1}^{(\alpha)}, u_{2}^{(\alpha)}\right)$ is not provided because it is not needed to derive the macro-scale equations. Introducing the auxiliary unknown

this can be rewritten as

$$
\widetilde{C}_{k, 1}^{(\alpha)}=C_{k, 1}^{(\alpha)}+\frac{q z_{k} C_{k, 0}^{(\alpha)}}{k_{B} T} u_{1}^{(\alpha)}
$$

$$
\underline{J}_{k, 0}^{(\alpha)}=-D_{k}\left(\underline{\underline{F}}^{T} \cdot \underline{\underline{F}}\right)^{-1} \cdot\left(J \underline{\nabla}_{\underline{\xi}}\left(J^{-1} C_{k, 0}^{(\alpha)}\right)+\frac{q z_{k} C_{k, 0}^{(\alpha)}}{k_{B} T} \underline{\nabla}_{\underline{\xi}} u_{0}^{(\alpha)}+\underline{\nabla}_{\underline{\eta}} \widetilde{C}_{k, 1}^{(\alpha)}\right) .
$$

Next, using expansions of the fluxes and concentrations, we can derive an expansion of (21),

$$
\partial_{t} C_{k, 0}^{(\alpha)}+\varepsilon \partial_{t} C_{k, 1}^{(\alpha)}=-\frac{1}{\varepsilon} \underline{\nabla}_{\underline{\eta}} \cdot \underline{J}_{k, 0}^{(\alpha)}-\underline{\nabla}_{\underline{\xi}} \cdot \underline{J}_{k, 0}^{(\alpha)}-\underline{\nabla}_{\underline{\eta}} \cdot \underline{J}_{k, 1}^{(\alpha)}-\varepsilon \underline{\nabla}_{\underline{\xi}} \cdot \underline{J}_{k, 1}^{(\alpha)}+o(\varepsilon),
$$

from which we can infer a cascade of equations by equating the coefficients of the successive powers of $\varepsilon$. For the coefficient of $\varepsilon^{-1}$, we get

$$
\underline{\nabla}_{\underline{\eta}} \cdot \underline{J}_{k, 0}^{(\alpha)}=0
$$


This is an equation set on $Y^{(\alpha)}$ in which the macroscopic variable $\xi$ and the time $t$ can be considered as parameters. Corresponding boundary conditions can be obtained by using (23), for which the term of degree 0 in $\varepsilon$ gives, recalling (32) and (34),

$$
\underline{J}_{k, 0}^{(\alpha)} \cdot \underline{n}_{0}^{(\alpha)}=0, \quad \text { on } \Gamma_{0}
$$

which can be rephrased as

$$
\underline{J}_{k, 0}^{(\alpha)} \cdot \underline{n}_{Y}^{(\alpha)}=0, \quad \text { on } \Gamma_{Y}
$$

since $\underline{n}_{0}^{(\alpha)}$ only depends on the micro-structure variable $\eta$.

Gathering (37), (39) and (40), we have the following complete system of equations for $\widetilde{C}_{k, 1}^{(\alpha)}$

$$
\begin{cases}\underline{\nabla}_{\underline{\eta}} \cdot\left[\left(\underline{\underline{F}}^{T} \cdot \underline{\underline{F}}\right)^{-1} \cdot \underline{\nabla}_{\underline{\eta}} \widetilde{C}_{k, 1}^{(\alpha)}\right]=0, & \text { in } Y^{(\alpha)} \\ {\left[\left(\underline{\underline{F}}^{T} \cdot \underline{\underline{F}}\right)^{-1} \cdot\left(\underline{\nabla}_{\underline{\eta}} \widetilde{C}_{k, 1}^{(\alpha)}+J \underline{\nabla}_{\underline{\xi}}\left(J^{-1} C_{k, 0}^{(\alpha)}\right)+\frac{q z_{k} C_{k, 0}^{(\alpha)}}{k_{B} T} \underline{\nabla}_{\underline{\xi}} u_{0}^{(\alpha)}\right)\right] \cdot \underline{n}_{Y}^{(\alpha)}=0 .} & \text { on } \Gamma_{Y}\end{cases}
$$

This should be understood as a set of partial differential equations in $\underline{\eta}$ parametrized by $\underline{\xi}$ and time. At this stage, it is possible to solve this so-called cell problem to obtain an expression of $\widetilde{C}_{k, 1}^{(\alpha)}$ as a function of the leading terms of the Ansatz. To that purpose we introduce the following basic cell problem in which, for any vector $\underline{v} \in \mathbb{R}^{3}$, we call $\mathcal{X}^{(\alpha)}(\underline{v})$ the periodic solution (defined up to a constant) of

$$
\left\{\begin{array}{cc}
\underline{\nabla}_{\underline{\eta}} \cdot\left[\left(\underline{\underline{F}}^{T} \cdot \underline{\underline{F}}\right)^{-1} \cdot \underline{\nabla}_{\underline{\eta}} \mathcal{X}^{(\alpha)}(\underline{v})\right]=0, & \text { in } Y^{(\alpha)} \\
{\left[\left(\underline{\underline{F}}^{T} \cdot \underline{\underline{F}}\right)^{-1} \cdot \underline{\nabla}_{\underline{\eta}} \mathcal{X}^{(\alpha)}(\underline{v})\right] \cdot \underline{n}_{Y}^{(\alpha)}=-\left[\left(\underline{\underline{F}}^{T} \cdot \underline{\underline{F}}\right)^{-1} \underline{v}\right] \cdot \underline{n}_{Y}^{(\alpha)},} & \text { on } \Gamma_{Y}
\end{array}\right.
$$

Noting that $\left(\underline{\underline{F}}^{T} \cdot \underline{\underline{F}}\right)^{-1}$ is a positive definite tensor, $\mathcal{X}^{(\alpha)}(\underline{v})$ is the well-defined solution of an elliptic problem in $\underline{\eta}$, at any point $\underline{\xi}$ in $\Omega_{0}$ and any time $t$, and for any choice of $\underline{v}$. Finally, we infer

$$
\widetilde{C}_{k, 1}^{(\alpha)}=\mathcal{X}^{(\alpha)}\left(J \underline{\nabla}_{\underline{\xi}}\left(J^{-1} C_{k, 0}^{(\alpha)}\right)+\frac{q z_{k} C_{k, 0}^{(\alpha)}}{k_{B} T} \underline{\nabla}_{\underline{\xi}} u_{0}^{(\alpha)}\right),
$$

and we can substitute this expression into (37) to obtain

$$
\begin{aligned}
\underline{J}_{k, 0}^{(\alpha)}=-J D_{k}\left(\underline{\underline{F}}^{T} \cdot \underline{\underline{F}}\right)^{-1} \cdot\left[\underline{\nabla}_{\underline{\xi}}\left(J^{-1} C_{k, 0}^{(\alpha)}\right)\right. & +\underline{\nabla}_{\underline{\eta}} \mathcal{X}^{(\alpha)}\left(\underline{\nabla}_{\underline{\xi}}\left(J^{-1} C_{k, 0}^{(\alpha)}\right)\right) \\
& \left.+\frac{q z_{k} J^{-1} C_{k, 0}^{(\alpha)}}{k_{B} T}\left(\underline{\nabla}_{\underline{\xi}} u_{0}^{(\alpha)}+\underline{\nabla}_{\underline{\eta}} \mathcal{X}^{(\alpha)}\left(\underline{\nabla}_{\underline{\xi}} u_{0}^{(\alpha)}\right)\right)\right],
\end{aligned}
$$

where we have used the (obvious) linearity of $\mathcal{X}^{(\alpha)}(\underline{v})$ with respect to its argument $\underline{v}$.

\subsubsection{Macro-scale equations for ionic concentrations}

In this section, we derive the macroscopic equations satisfied by the leading terms of our expansions. Identifying terms of order 0 in (38), we get

$$
\frac{\partial C_{k, 0}^{(\alpha)}}{\partial t}+\underline{\nabla}_{\underline{\xi}} \cdot \underline{J}_{k, 0}^{(\alpha)}+\underline{\nabla}_{\underline{\eta}} \cdot \underline{J}_{k, 1}^{(\alpha)}=0 .
$$


The macro-scale equation is then obtained by integrating (44) with respect to $\eta$ over $Y^{(\alpha)}$. Using the Stokes formula on the last term, we get

$$
\left|Y^{(\alpha)}\right| \frac{\partial C_{k, 0}^{(\alpha)}}{\partial t}+\underline{\nabla}_{\underline{\xi}} \cdot\left|Y^{(\alpha)}\right| \underline{\widehat{J}}_{k, 0}^{(\alpha)}+\int_{\Gamma_{Y}} \underline{J}_{k, 1}^{(\alpha)} \cdot \underline{n}_{Y}^{(\alpha)} d \Gamma=0,
$$

where we have introduced the average flux quantities

$$
\underline{\widehat{J}}_{k, 0}^{(\alpha)}=\frac{1}{\left|Y^{(\alpha)}\right|} \int_{Y^{(\alpha)}} \underline{J}_{k, 0}^{(\alpha)} d \underline{\eta} .
$$

It is classical in periodic homogenization analysis (see e.g. [3, Chapter 1]) to introduce the tensor $\underline{\underline{T}}^{(\alpha)}$ defined by

$$
\underline{\underline{T}}^{(\alpha)} \cdot \underline{u}=\frac{1}{\left|Y^{(\alpha)}\right|} \int_{Y^{(\alpha)}}\left(\underline{\underline{F}}^{T} \cdot \underline{\underline{F}}\right)^{-1} \cdot\left(\underline{u}+\underline{\nabla}_{\underline{\eta}} \mathcal{X}^{(\alpha)}(\underline{u})\right) d \underline{\eta} .
$$

Note that $\underline{\underline{T}}^{(\alpha)}$ only depends on the geometry of the cell and on the deformation (through $\underline{\underline{F}}$ ).

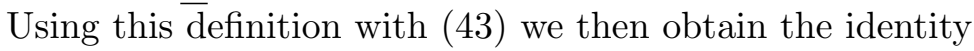

$$
\underline{\widehat{J}}_{k, 0}^{(\alpha)}=-J D_{k} \underline{\underline{T}}^{(\alpha)} \cdot\left(\underline{\nabla}_{\underline{\xi}}\left(J^{-1} C_{k, 0}^{(\alpha)}\right)+\frac{q z_{k} J^{-1} C_{k, 0}^{(\alpha)}}{k_{B} T} \underline{\nabla}_{\underline{\xi}} u_{0}^{(\alpha)}\right) .
$$

Remark 1 Using the weak form of (41), the following equivalent expression for $\underline{\underline{T}}^{(\alpha)}$ can be derived

$$
\left(\underline{\underline{T}}^{(\alpha)} \cdot \underline{u}\right) \cdot \underline{v}=\frac{1}{\left|Y^{(\alpha)}\right|} \int_{Y^{(\alpha)}}\left(\left(\underline{\underline{F}}^{T} \cdot \underline{F}\right)^{-1} \cdot\left(\underline{u}+\underline{\nabla}_{\underline{\eta}} \mathcal{X}^{(\alpha)}(\underline{u})\right)\right) \cdot\left(\underline{v}+\underline{\nabla}_{\underline{\eta}} \mathcal{X}^{(\alpha)}(\underline{v})\right) d \underline{\eta},
$$

which shows that $\underline{\underline{T}}^{(\alpha)}$ is symmetric definite positive. Note that the definite property can be obtained as follows

$$
\left(\underline{\underline{T}}^{(\alpha)} \cdot \underline{u}\right) \cdot \underline{u}=0 \quad \Rightarrow \quad \underline{u}+\underline{\nabla}_{\underline{\eta}} \mathcal{X}^{(\alpha)}(\underline{u})=0,
$$

which implies $\underline{u}=0$, since $\underline{\nabla}_{\underline{\eta}} \mathcal{X}^{(\alpha)}(\underline{u})$ cannot be constant and non-zero due to the periodicity of $\mathcal{X}^{(\alpha)}$, see [3] for more detail.

To conclude, we need to derive boundary conditions for $\underline{J}_{k, 1}^{(\alpha)} \cdot \underline{n}_{Y}^{(\alpha)}$. These conditions are obtained by first substituting the Ansatz in the terms defined on the membrane $\Gamma_{0}$ in (23), and then identifying terms proportional to $\varepsilon$. We get

$$
\left\{\begin{array}{l}
\underline{J}_{k, 1}^{(i)} \cdot \underline{n}_{Y}^{(i)}=\frac{\partial \mu_{k, 0}^{(i)}}{\partial t}+I_{k, 0}^{i o n} \\
\underline{J}_{k, 1}^{(e)} \cdot \underline{n}_{Y}^{(e)}=\frac{\partial \mu_{k, 0}^{(e)}}{\partial t}-I_{k, 0}^{i o n}
\end{array}\right.
$$

Since by assumption - recall (33) and (34) - the terms $\mu_{k, 0}^{(\alpha)}$ and $I_{k, 0}^{i o n}$ are independent of $\eta$ we get from (49)

$$
\left\{\begin{array}{l}
\frac{1}{|Y|} \int_{\Gamma_{Y}} \underline{J}_{k, 1}^{(i)} \cdot \underline{n}_{Y}^{(i)}=A_{m}\left(\frac{\partial \mu_{k, 0}^{(i)}}{\partial t}+I_{k, 0}^{i o n}\right) \\
\frac{1}{|Y|} \int_{\Gamma_{Y}} \underline{J}_{k, 1}^{(e)} \cdot \underline{n}_{Y}^{(e)}=A_{m}\left(\frac{\partial \mu_{k, 0}^{(e)}}{\partial t}-I_{k, 0}^{i o n}\right)
\end{array}\right.
$$

defining the quantity $A_{m}=\frac{\left|\Gamma_{Y}\right|}{|Y|}$, i.e. the ratio of membrane area to overall volume in the reference cell. 


\subsection{Summary of equations in Lagrangian framework}

Defining the volume fractions of intra- and extra-cellular media $\phi^{(\alpha)}$ in the reference configuration as

$$
\phi^{(\alpha)}=\frac{\left|Y^{(\alpha)}\right|}{|Y|},
$$

we divide (45) by $|Y|$ and gather the result with (47) and (50) as follows

$$
\left\{\begin{array}{l}
\phi^{(\alpha)} \frac{\partial C_{k, 0}^{(\alpha)}}{\partial t}+\underline{\nabla}_{\underline{\xi}} \cdot \phi^{(\alpha)} \underline{\widehat{J}}_{k, 0}^{(\alpha)}+A_{m} p_{k, 0}^{(\alpha)}=0 \\
\underline{\widehat{J}}_{k, 0}^{(\alpha)}=-J D_{k} \underline{\underline{T}}^{(\alpha)} \cdot\left(\underline{\nabla}_{\underline{\xi}}\left(J^{-1} C_{k, 0}^{(\alpha)}\right)+\frac{q z_{k}\left(J^{-1} C_{k, 0}^{(\alpha)}\right)}{k_{B} T} \underline{\nabla}_{\underline{\xi}} u_{0}^{(\alpha)}\right),
\end{array}\right.
$$

with

$$
\left\{\begin{array}{l}
p_{k, 0}^{(i)}=\frac{\partial \mu_{k, 0}^{(i)}}{\partial t}+I_{k, 0}^{i o n} \\
p_{k, 0}^{(e)}=\frac{\partial \mu_{k, 0}^{(e)}}{\partial t}-I_{k, 0}^{i o n}
\end{array}\right.
$$

and the additional relations pertaining to surface charges read - recalling (31) and (33)

$$
\left\{\begin{array}{l}
\mu_{k, 0}^{(\alpha)}=\frac{z_{k} C_{k, 0}^{(\alpha)}}{\sum_{\ell}\left(z_{\ell}\right)^{2} C_{\ell, 0}^{(\alpha)}} \frac{\mu_{0}^{(\alpha)}}{\mathrm{F}} \\
\mu_{0}^{(i)}=-\mu_{0}^{(e)}=\widehat{C}_{m} V_{m, 0}
\end{array}\right.
$$

Note that the terms $A_{m} p_{k, 0}^{(\alpha)}$ in (51) represent the quantity of ions going from the volume to the membrane, for each species and in each domain (per unit volume of the reference configuration). Finally, we complete this set of equations by the homogenized version of (24). Following the same ideas as in the previous sections, one can show that

$$
\left\{\begin{array}{l}
\underline{\nabla}_{\underline{\xi}} \cdot \phi^{(\alpha)} \underline{\widehat{J}}_{0}^{(\alpha)}+A_{m} p_{0}^{(\alpha)}=0 \\
\underline{\widehat{J}}_{0}^{(\alpha)}=-J \underline{\underline{T}}^{(\alpha)} \cdot\left(\sigma_{0}^{(\alpha)} \underline{\nabla}_{\underline{\xi}} u_{0}^{(\alpha)}+\mathrm{F} \sum_{k=1}^{N^{(\alpha)}} z_{k} D_{k} \underline{\nabla}_{\underline{\xi}}\left(J^{-1} C_{k, 0}^{(\alpha)}\right)\right),
\end{array}\right.
$$

with

$$
\left\{\begin{array}{l}
p_{0}^{(i)}=-p_{0}^{(e)}=\widehat{C}_{m} \frac{\partial V_{m, 0}}{\partial t}+I_{t o t, 0}^{i o n} \\
\sigma_{0}^{(\alpha)}=\mathrm{F} q \sum_{k=1}^{N^{(\alpha)}} m_{k}\left(z_{k}\right)^{2} J^{-1} C_{k, 0}^{(\alpha)}
\end{array}\right.
$$

where

$$
I_{\text {tot }, 0}^{i o n}=\mathrm{F} \sum_{k=1}^{N} z_{k} I_{k, 0}^{i o n} .
$$

Remark 2 The homogenized equations obtained above can be shown to also hold in the framework of so-called locally periodic media. We refer to [58] for a mathematical definition and 
treatment of locally periodic homogenization by 2-scale convergence. This framework is particularly well-suited to the modeling of the heart because of its fiber structure of smoothly varying orientation, see Figure 4, and we refer e.g. to [8] for a detailed mathematical description of this structure. In terms of homogenization this corresponds to assuming that the cell geometry $Y$ is a regular function of $\underline{\xi}$ with "slow variations", see [58] for a precise definition. Applying these concepts, the above homogenized equations (51)-(54) are exactly preserved, up to the fact that $A_{m},\left|Y^{(\alpha)}\right|,|Y|$ and $\underline{\underline{T}}^{(\alpha)}$ are now functions of $\underline{\xi}$.

Bidomain simplifications. Assuming that the diffusion is negligible compared to the drift, one can simplify System (54) into

$$
\left\{\begin{array}{l}
\underline{\nabla}_{\underline{\xi}} \cdot\left(\underline{\underline{\Sigma}}_{0}^{(i)} \cdot \underline{\nabla}_{\underline{\xi}} u_{0}^{(i)}+\underline{\underline{\Sigma}}_{0}^{(e)} \cdot \underline{\nabla}_{\underline{\xi}} u_{0}^{(e)}\right)=0, \\
A_{m}\left[\widehat{C}_{m} \frac{\partial V_{m, 0}}{\partial t}+I_{t o t, 0}^{i o n}\right]-\underline{\nabla}_{\underline{\xi}} \cdot\left(\underline{\underline{\Sigma}}_{0}^{(i)} \cdot \underline{\nabla}_{\underline{\xi}} u_{0}^{(i)}\right)=0,
\end{array}\right.
$$

with

$$
\underline{\underline{\Sigma}}_{0}^{(\alpha)}=J \sigma_{0}^{(\alpha)} \phi^{(\alpha)} \underline{\underline{T}}^{(\alpha)}
$$

\subsection{Summary of equations in Eulerian framework}

We can obtain the equivalent Eulerian form of the homogenized Equations (51) and (53). Defining

$$
c_{k, 0}^{(\alpha)}=J^{-1} C_{k, 0}^{(\alpha)}, \quad \underline{\widehat{J}}_{k, r 0}^{(\alpha)}=J^{-1} \underline{\underline{F}} \cdot \underline{\widehat{J}}_{k, 0}^{(\alpha)}, \quad \underline{\widehat{\jmath}}_{k, 0}^{(\alpha)}=\underline{\widehat{\jmath}}_{k, r 0}^{(\alpha)}+c_{k, 0}^{(\alpha)} \underline{v}_{s},
$$

and the Eulerian tensor $\underline{\underline{T}}_{E}^{(\alpha)}$ as

$$
\underline{\underline{T}}_{E}^{(\alpha)}=\underline{\underline{F}} \cdot \underline{\underline{T}}^{(\alpha)} \cdot \underline{\underline{F}}^{T}
$$

we directly infer

$$
\left\{\begin{array}{l}
\left.\frac{\partial\left(\phi^{(\alpha)} c_{k, 0}^{(\alpha)}\right)}{\partial t}\right|_{\underline{x}}+\underline{\nabla}_{\underline{x}} \cdot\left(\phi^{(\alpha)} \underline{\hat{\jmath}}_{k, 0}^{(\alpha)}\right)+J^{-1} A_{m} p_{k, 0}^{(\alpha)}=0 \\
\underline{\hat{\jmath}}_{k, r 0}^{(\alpha)}=-D_{k} \underline{T}_{E}^{(\alpha)} \cdot\left(\underline{\nabla}_{\underline{x}} c_{k, 0}^{(\alpha)}+\frac{q z_{k} c_{k, 0}^{(\alpha)}}{k_{B} T} \underline{\nabla}_{\underline{x}} u_{0}^{(\alpha)}\right)
\end{array}\right.
$$

while the additional equations (52) and (53) remain unchanged, up to the use of Eulerian - i.e. physical - concentrations in the expressions, viz.

$$
\begin{gathered}
\left\{\begin{array}{l}
p_{k, 0}^{(i)}=\left.\frac{\partial \mu_{k, 0}^{(i)}}{\partial t}\right|_{\underline{\xi}}+I_{k, 0}^{i o n}, \\
p_{k, 0}^{(e)}=\left.\frac{\partial \mu_{k, 0}^{(e)}}{\partial t}\right|_{\underline{\xi}}-I_{k, 0}^{i o n},
\end{array}\right. \\
\left\{\begin{array}{l}
\mu_{k, 0}^{(\alpha)}=\frac{z_{k} c_{k, 0}^{(\alpha)}}{\sum_{\ell}\left(z_{\ell}\right)^{2} c_{\ell, 0}^{(\alpha)}} \frac{\mu_{0}^{(\alpha)}}{\mathrm{F}}, \\
\mu_{0}^{(i)}=-\mu_{0}^{(e)}=\widehat{C}_{m} V_{m, 0} .
\end{array}\right.
\end{gathered}
$$

Likewise, defining

$$
\underline{\widehat{J}}_{r 0}^{(\alpha)}=J^{-1} \underline{\underline{F}} \cdot \underline{\widehat{J}}_{0}^{(\alpha)},
$$


we have

$$
\left\{\begin{array}{l}
\underline{\nabla}_{\underline{x}} \cdot\left(\phi^{(\alpha)} \underline{\widehat{\jmath}}_{r 0}^{(\alpha)}\right)+J^{-1} A_{m} p_{0}^{(\alpha)}=0 \\
\underline{\hat{\jmath}}_{r 0}^{(\alpha)}=-\underline{T}_{E}^{(\alpha)} \cdot\left(\sigma_{0}^{(\alpha)} \underline{\nabla}_{\underline{x}} u_{0}^{(\alpha)}+\mathrm{F} \sum_{k=1}^{N^{(\alpha)}} z_{k} D_{k} \underline{\nabla}_{\underline{x}} c_{k, 0}^{(\alpha)}\right)
\end{array}\right.
$$

with

$$
\left\{\begin{array}{l}
p_{0}^{(i)}=-p_{0}^{(e)}=\left.\widehat{C}_{m} \frac{\partial V_{m, 0}}{\partial t}\right|_{\underline{\xi}}+I_{\text {tot }, 0}^{i o n} \\
\sigma_{0}^{(\alpha)}=\mathrm{F} q \sum_{k=1}^{N^{(\alpha)}} m_{k}\left(z_{k}\right)^{2} c_{k, 0}^{(\alpha)}
\end{array}\right.
$$

Bidomain model simplifications. Neglecting the diffusion effect in (62), we obtain

$$
\left\{\begin{array}{l}
\underline{\nabla}_{\underline{x}} \cdot\left(\underline{\underline{\sigma}}^{(i)} \cdot \underline{\nabla}_{\underline{x}} u_{0}^{(i)}+\underline{\underline{\sigma}}^{(e)} \cdot \underline{\nabla}_{\underline{x}} u_{0}^{(e)}\right)=0, \\
J^{-1} A_{m}\left[\left.\widehat{C}_{m} \frac{\partial V_{m, 0}}{\partial t}\right|_{\underline{\xi}}+I_{t o t, 0}^{i o n}\right]-\underline{\nabla}_{\underline{x}} \cdot\left(\underline{\underline{\sigma}}^{(i)} \cdot \underline{\nabla}_{\underline{x}} u_{0}^{(i)}\right)=0,
\end{array}\right.
$$

with $\underline{\underline{\sigma}}^{(\alpha)}=\phi^{(\alpha)} \sigma_{0}^{(\alpha)} \underline{\underline{T}}_{E}^{(\alpha)}$.

Eulerian cell problem for homogenized tensor. We have, recalling (46),

$$
\begin{aligned}
\left(\underline{\underline{T}}_{E}^{(\alpha)} \cdot \underline{u}\right) \cdot \underline{v}=\left(\underline{\underline{T}}^{(\alpha)} \cdot\left(\underline{\underline{F}}^{T} \cdot \underline{u}\right)\right) \cdot\left(\underline{\underline{F}}^{T} \cdot \underline{v}\right) & =\frac{1}{\left|Y^{(\alpha)}\right|} \int_{Y^{(\alpha)}}\left(\underline{u}+\underline{\underline{F}}^{-T} \cdot \underline{\nabla}_{\underline{\eta}} \mathcal{X}^{(\alpha)}\left(\underline{\underline{F}}^{T} \cdot \underline{u}\right)\right) \cdot \underline{v} d \underline{\eta} \\
& =\frac{1}{\left|\underline{\phi}\left(Y^{(\alpha)}\right)\right|} \int_{\underline{\phi}\left(Y^{(\alpha)}\right)}\left(\underline{u}+\underline{\nabla}_{\underline{y}} \mathcal{X}_{\phi}^{(\alpha)}(\underline{u})\right) \cdot \underline{v} d \underline{y}, \quad(65)
\end{aligned}
$$

where we denote $\mathcal{X}_{\phi}^{(\alpha)}(\underline{u})=\mathcal{X}^{(\alpha)}\left(\underline{\underline{F}}^{T} \cdot \underline{u}\right)$. Moreover, we note that the equivalent variational form for the cell problem $(41)$ is: Find $\mathcal{X}^{(\alpha)}(\underline{v})$ periodic such that, for all periodic $\mathcal{X}$,

$$
\int_{Y^{(\alpha)}}\left(\left(\underline{\underline{F}}^{T} \cdot \underline{\underline{F}}\right)^{-1} \cdot\left(\underline{\nabla}_{\underline{\eta}} \mathcal{X}^{(\alpha)}(\underline{v})+\underline{v}\right)\right) \cdot \underline{\nabla}_{\underline{\eta}} \mathcal{X} d \underline{\eta}=0 .
$$

Applying this for $\underline{v}=\underline{F}^{T} \cdot \underline{u}$ and changing variables via the mapping $\underline{\phi}$ we obtain equivalently

$$
\int_{\underline{\phi}\left(Y^{(\alpha)}\right)}\left(\underline{\nabla}_{\underline{y}} \mathcal{X}_{\phi}^{(\alpha)}(\underline{u})+\underline{u}\right) \cdot \underline{\nabla}_{\underline{y}} \mathcal{X} d \underline{y}=0
$$

which shows that the homogenized tensor in the Eulerian framework can be obtained through a natural cell problem posed in the deformed configuration, i.e. the form that would be obtained with (66) in the absence of deformation $(\underline{F}=\underline{I})$ albeit posed here in the deformed cell. This was to be expected since the deformation is assumed to be slowly varying, and therefore satisfies the local periodicity assumption already discussed in Remark 2.

Remark 3 The tensors $\underline{\underline{T}}_{E}^{(\alpha)}$ and $\underline{\underline{T}}^{(\alpha)}$ - that directly provide the conductivity tensors in the homogenized equations - are obtained by solving cell problems that depend on the deformation - at the point considered - via the deformation gradient $\underline{\underline{F}}$. Characterizing this dependence more precisely is of course an interesting question. We point out that there does not appear to be a direct transformation rule from the tensors in the undeformed configuration $(\underline{\underline{F}}=\underline{\underline{I}})$ to 
those in a deformed one. Nevertheless, we have the property - immediately seen from (41) and (46) - that $\underline{\underline{T}}^{(\alpha)}$ only depends on the deformation via $\underline{\underline{F}}^{T} \cdot \underline{\underline{F}}$, i.e. the so-called right CauchyGreen tensor, which directly implies that the Lagrangian tensor is invariant in all rigid body deformations, as could be expected. We will further discuss the dependence on the deformation in the case of cardiac cells in the next section.

\section{$4 \quad$ Numerical illustrations}

In this section, we will perform a detailed numerical assessment of a simplified model derived based on the proposed bidomain model (64). First we present the simplified model obtained by assuming a closed-form expression for the tensor $\underline{\underline{T}}_{E}^{(\alpha)}$, and we substantiate this assumption by numerically comparing the resulting tensors with those obtained by solving the above-described cell problems. Secondly, we present the realistic cardiac electrophysiology context and the numerical methods that we have used to perform the simulations. In a third part, we compare a complete simulation taking into account the mechanical deformations with a simulation where we neglect them. Finally, we illustrate the impact of mechanical deformations on the simulation of electrocardiograms.

\subsection{Construction of an approximate homogenized conductivity tensor in de- formed configuration}

The exact computation of the homogenized conductivity tensor would rely on the a priori description of a typical cell geometry for each point of the reference configuration $\Omega_{0}$. Then the canonical cell problems (41) should be solved at each of these points and for every given deformation tensor $\underline{F}$. This makes for a complex procedure in which, moreover, a detailed description of the cell geometry is out of reach in practice, and therefore rather artificial. As a consequence we look for alternative strategies to build the conductivity tensors, albeit in such a way that the essential features of these tensors - and of the effect of mechanical deformations - are reasonably captured.

First, let us assume that the periodicity cell at any point in the tissue $Y(\underline{\xi})$ is obtained by a rotation of a reference periodicity cell $\hat{Y}$ given by

$$
\hat{Y}=\left[-\frac{L}{2}, \frac{L}{2}\right] \times\left[-\frac{H}{2}, \frac{H}{2}\right]^{2},
$$

so that the first direction is aligned with the fiber direction. In practice, typical values for the aspect ratio $L / H$ are in the range $5-10$. We also assume that the geometry is invariant by rotations of 90 degrees around this first direction. This implies that the resulting tensor $\underline{\underline{T}}^{(\alpha)}$ is transversely-isotropic when $\underline{\underline{F}}=\underline{\underline{I}}$, as often assumed in cardiac electrophysiology, i.e.

$$
\underline{\underline{T}}^{(\alpha)}=c_{t}^{(\alpha)} \underline{\underline{I}}+\left(c_{l}^{(\alpha)}-c_{t}^{(\alpha)}\right) \underline{\tau}_{0} \otimes \underline{\tau}_{0},
$$

where $\underline{\tau}_{0}$ is a unit vector associated with the fiber direction at any point, and with the longitudinal and transverse scalar coefficients $c_{l}^{(\alpha)}$ and $c_{t}^{(\alpha)}$ (respectively) independent of the point considered. When deformations are considered, it is then natural to evaluate how the homogenized tensor differs from one associated with the same scalar coefficients, albeit with the new fiber direction $\underline{\tau}=\frac{\underline{\underline{F} \cdot \underline{\tau}_{0}}}{\left\|\underline{\underline{\underline{F}}} \cdot \underline{\underline{\tau}}_{0}\right\|}$, i.e.

$$
\underline{\underline{T}}_{E}^{(\alpha)}=c_{t}^{(\alpha)} \underline{\underline{I}}+\left(c_{l}^{(\alpha)}-c_{t}^{(\alpha)}\right) \underline{\tau} \otimes \underline{\tau},
$$


or equivalently in Lagrangian form

$$
\underline{\underline{\tilde{T}}}^{(\alpha)}=\underline{\underline{F}}^{-1} \cdot \underline{\underline{\tilde{T}}}_{E}^{(\alpha)} \cdot \underline{\underline{F}}^{-T}=c_{t}^{(\alpha)}\left(\underline{\underline{F}}^{T} \cdot \underline{\underline{F}}\right)^{-1}+\left(c_{l}^{(\alpha)}-c_{t}^{(\alpha)}\right)\left(\left\|\underline{\underline{F}} \cdot \underline{\tau}_{0}\right\|^{-1} \underline{\tau}_{0}\right) \otimes\left(\left\|\underline{\underline{F}} \cdot \underline{\tau}_{0}\right\|^{-1} \underline{\tau}_{0}\right) .
$$

Note that this expression only depends on $\underline{\underline{F}}$ via $\underline{\underline{F}}^{T} \cdot \underline{\underline{F}}-$ i.e. the right Cauchy-Green deformation tensor - as it should be.

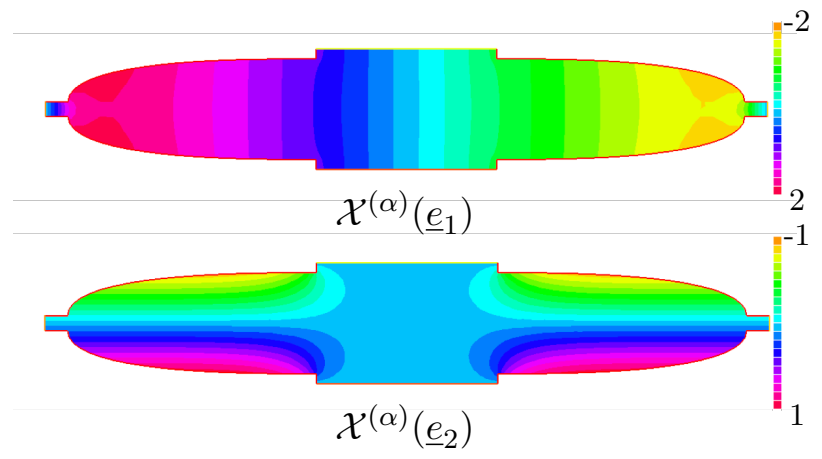

(a) $\underline{\underline{F}}=\underline{\underline{I}}$

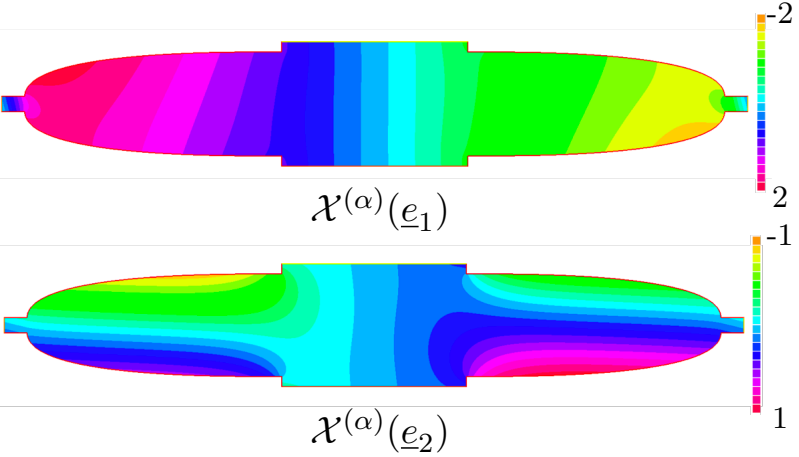

(b) $\underline{\underline{F}}=\underline{\underline{I}}+\underline{e}_{1} \otimes \underline{e}_{2} / 5$

Figure 2: Geometry of 2D periodicity cell, and computed function $\mathcal{X}^{(\alpha)}$ for undeformed and sheared problems.

Our objective is now to compare the tensors provided by the closed-form expressions (68)(69) with those obtained by numerically solving the cell homogenization problem for a representative range of deformations $\underline{\underline{F}}$. As an example, we consider the $2 \mathrm{D}$ cell shown in Figure 2 for which we focus on the intracellular region - as the connectivity of the extracellular space cannot be made realistic in 2D as the intracellular region constitutes a barrier - and we use FreeFem++ to compute finite element approximations of the cell problems (41) on a very fine mesh [28]. More precisely, Eqs. (46) and (65) are used for the computation of tensors in the Lagrangian and Eulerian frameworks, respectively. The initial computation for the undeformed problem provides the scalar coefficients $c_{l}^{(\alpha)}$ and $c_{t}^{(\alpha)}$, and we then use these values in (68)(69) to compute the error $\underline{\underline{T}}^{(\alpha)}-\underline{\underline{\underline{T}}}^{(\alpha)}$ when varying the deformation $\underline{\underline{F}}$ - here considered as incompressible, i.e. $\operatorname{det} \underline{\underline{F}}=1$, a common assumption in biological tissues - see the results in Figure 3. We observe that the relative error does not exceed $4 \%$ for deformations of up to $20 \%$, and that the largest errors are reached for deformations involving large shear, to an extent that is unlikely to be frequently encountered in practice. We also display in Figure 2 the computed functions $\mathcal{X}^{(\alpha)}\left(\underline{e}_{1}\right)$ and $\mathcal{X}^{(\alpha)}\left(\underline{e}_{2}\right)$ for the undeformed problem and for that associated with the largest shear.

Finally, although we cannot draw definitive conclusions from this 2D numerical study, in our cardiac simulations we will approximate the tensors $\underline{\underline{T}}^{(\alpha)}$ and $\underline{\underline{T}}_{E}^{(\alpha)}$ by $\underline{\underline{T}}^{(\alpha)}$ and $\underline{\underline{T}}_{E}^{(\alpha)}$ in order to focus on other effects of deformations. Denoting $\sigma_{t}^{(\overline{\bar{\alpha})}}=\sigma^{(\alpha)} c_{t}^{(\alpha)}$ and $\sigma_{l}^{(\alpha)}=\sigma^{(\alpha)} c_{l}^{(\alpha)}$, this amounts to approximating the Eulerian conductivity tensor as follows

$$
\underline{\underline{\sigma}}^{(\alpha)} \simeq \phi^{(\alpha)}\left[\sigma_{t}^{(\alpha)} \underline{\underline{I}}+\left(\sigma_{l}^{(\alpha)}-\sigma_{t}^{(\alpha)}\right) \underline{\tau} \otimes \underline{\tau}\right] .
$$

Note that this tensor depends on the deformation through the vector $\underline{\tau}$ that - by definition - represents the fiber direction in the deformed configuration. 


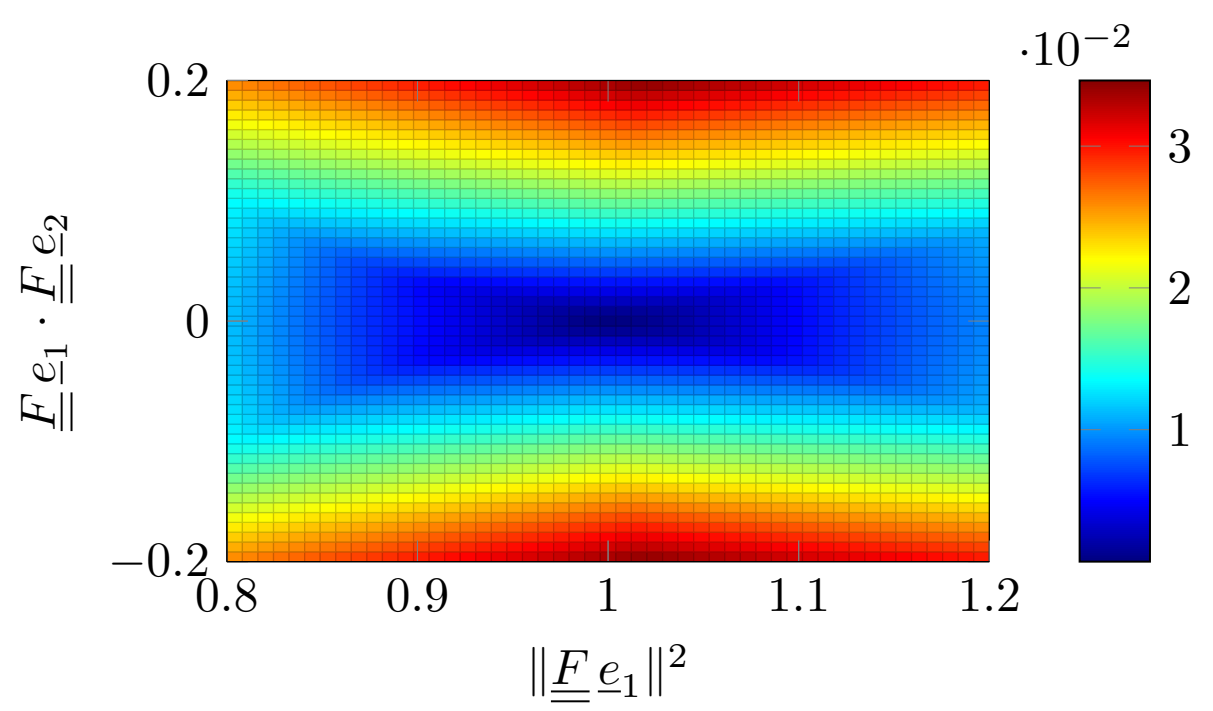

Figure 3: Relative matrix error $\left\|\underline{\underline{T}}^{(\alpha)}-\underline{\underline{\tilde{T}}}^{(\alpha)}\right\|_{\infty} /\left\|\underline{\underline{T}}^{(\alpha)}\right\|_{\infty}$ for varying $\underline{\underline{F}}($ with $\operatorname{det} \underline{\underline{F}}=1)$.

\begin{tabular}{cccc}
\hline \hline$\sigma_{t}^{(e)}$ & $\sigma_{l}^{(e)}$ & $\sigma_{t}^{(i)}$ & $\sigma_{l}^{(i)}$ \\
\hline \hline $8.010^{-4}$ & $2.210^{-3}$ & $3.010^{-4}$ & $2.210^{-3}$ \\
\hline
\end{tabular}

Table 1: Conductivity parameters (all in S.cm ${ }^{-1}$ ).

\subsection{Simulation results}

\subsubsection{Model description}

In our numerical simulations, we focus on the bidomain model given in (64). Dropping the "0" subscripts introduced in the definition of the homogenized quantities, these equations can be rewritten in a weak form as follows. For all $t>0$, find $V_{m}(\cdot, t) \in H^{1}(\Omega(t)), u^{(e)}(\cdot, t) \in H^{1}(\Omega(t))$ with $\int_{\Omega(t)} u^{(e)}=0$, such that

$$
\left\{\begin{array}{l}
\int_{\Omega(t)}\left(\underline{\underline{\sigma}}^{(i)} \cdot\left(\underline{\nabla}_{\underline{x}} V_{m}+\underline{\nabla}_{\underline{x}} u^{(e)}\right)\right) \cdot \underline{\nabla}_{\underline{x}} \phi d \Omega+\int_{\Omega_{0}} A_{m} \widehat{C}_{m}\left(\left.\frac{\partial V_{m}}{\partial t}\right|_{\underline{\xi}}+I_{t o t}^{i o n}\right) \phi d \Omega=0, \\
\int_{\Omega(t)} \underline{\underline{\sigma}}^{(i)} \cdot \underline{\nabla}_{\underline{x}} V_{m} \cdot \underline{\nabla}_{\underline{x}} \psi d \Omega+\int_{\Omega(t)}\left(\left(\underline{\underline{\sigma}}^{(e)}+\underline{\underline{\sigma}}^{(i)}\right) \cdot \underline{\nabla}_{\underline{x}} u^{(e)}\right) \cdot \underline{\nabla}_{\underline{x}} \psi d \Omega=0,
\end{array}\right.
$$

for all $\phi, \psi \in H^{1}(\Omega(t))$ such that

$$
\int_{\Omega(t)} \psi=0
$$

Note that the second integral term in the first equation of $(71)-i . e$. that inherited from the membrane equations - is posed in a natural manner in the reference configuration, as the time derivative of the transmembrane potential inside is intrinsically Lagrangian.

For the conductivity tensors $\underline{\sigma}^{(\alpha)}$, we use the approximate expression (70) instead of solving the homogenization cell problem. In so doing, we still expect to reasonably capture the effects of cell deformations on the macroscopic conductivity, as discussed in Section 4.1. This also indicates that conductivity is more naturally described in the deformed configuration, which is the reason why all the corresponding integrals of (71) are written in the deformed configuration. In our simulations we used the values of conductivity parameters given in Table 1 . These values are 

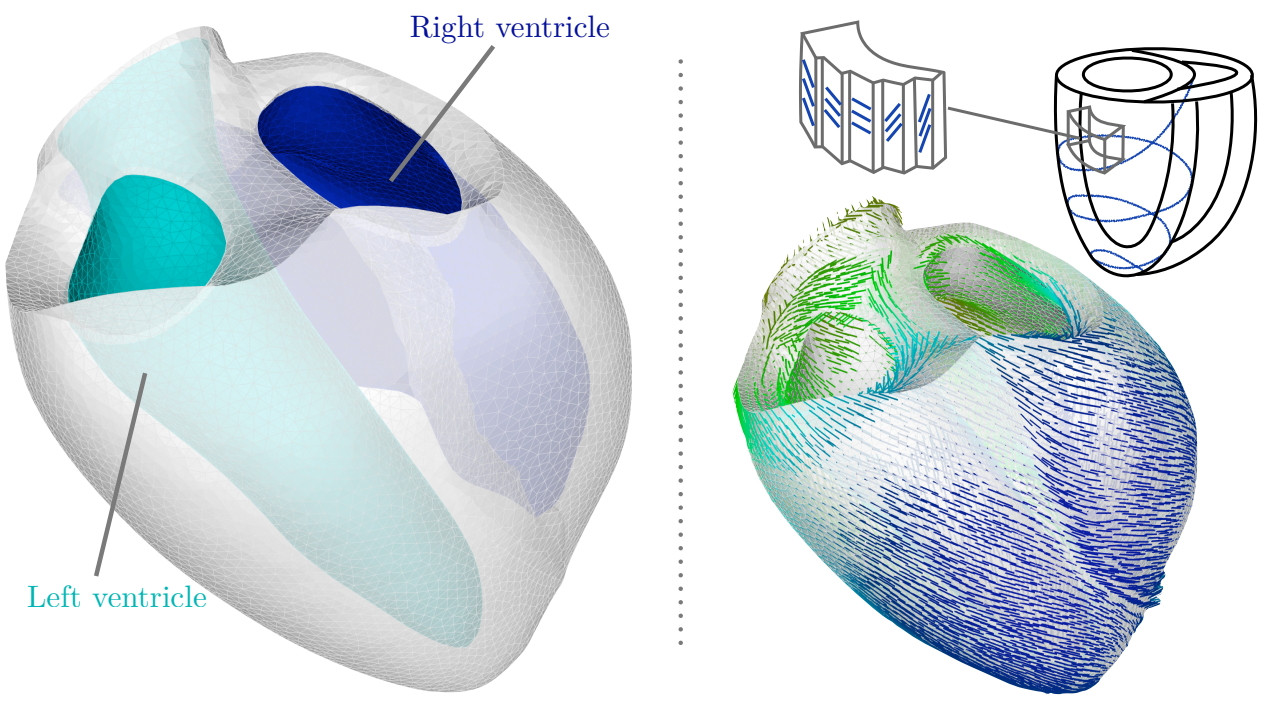

Figure 4: Left: Mesh of the ventricles. Right: Fibers in the ventricles.

close to those given in [54] and have been slightly adapted to obtain realistic electrocardiograms. The values of the membrane parameters are $A_{m}=200.0 \mathrm{~cm}^{-1}$ and $\widehat{C}_{m}=10^{-3} \mathrm{mF} . \mathrm{cm}^{-2}$.

As already mentioned, we will disregard the effect of deformations on ionic models discussed in Section 2.8, which means that we deliberately choose to focus on the other effects that have been little investigated before. In fact, we point out that the impact of writing the conductivities in the deformed configuration has already been studied in $[14,15,16,21,61]$, albeit without the Lagrangian time derivative that we have derived from our homogenization approach.

We will now describe the important ingredients required to set up and run simulations of this model in a realistic case.

Anatomical geometries First of all, in order to obtain patient-specific simulations, a geometry of the patient heart is needed. In the literature, many approaches are proposed using automatic (or semi-automatic) segmentation [31, 39, 78] to extract the contours of the epicardium and endocardium of the left and right ventricles of the heart. This allows to obtain a surface geometry of the patient mesh. In this paper, we use the Zygote ${ }^{1}$ heart model, a geometric model derived from anatomical data using segmentation.

Using the surface geometry of the patient mesh, a computational mesh adapted to finite elements was constructed. We used the 3-matic software to obtain a computationally correct surface mesh, and the Yams meshing software to refine the surface mesh [23]. Then, we meshed the volume of the two ventricles using Ghs3d [24] and Gmsh [25]. We can see in Figure 4 (left) the whole heart mesh, which contains about 230,600 tetrahedra.

We computed the cavity volume, the tissue mass and the valves diameters of the left ventricle of this mesh, and compared them with literature reports of normal human heart dimension indicators. Our results indicate that the geometry corresponds to ventricular end-systole, i.e. , when the ventricle chamber is smallest. This geometric configuration is the closest one - within a cardiac cycle - to the geometry associated with the stress-free reference configuration, so we directly use it as the reference configuration in our simulations.

Muscle fibers The fiber directions are needed to prescribe the anisotropic conductivity tensor given by (70). According to standard anatomical knowledge [47,68], it appears that in a good

\footnotetext{
${ }^{1}$ www.3dscience.com
} 
approximation the fiber angles vary from +70 to -70 degrees with respect to circumferential direction across the wall thickness from endo- to epicardium, see Figure 4 (right, top). Using an automatic method, we prescribe the fibers in the 3D mesh, see Figure 4 (right, bottom). This approximation strategy is very effective, especially since in-vivo measurement of fiber directions is still out of reach in clinical practice, although some promising research results are available in this direction $[45,67,74]$.

Ionic model As discussed in Section 2.8, many ionic models exist in the literature. In this paper, we present simulations performed with the Minimal model for human Ventricular action potentials model (MV model) [9]. This model has been adapted to the ventricular tissue including mid-myocardial, epicardial and endocardial cells. It is associated with three ionic currents, i.e. , $I_{t o t}^{i o n}=I_{f i}+I_{s o}+I_{s i}$, where

$$
\begin{aligned}
& I_{f i}=-H\left(V_{m}-\theta_{v}\right)\left(V_{m}-\theta_{v}\right)\left(V_{m}^{u}-V_{m}\right) \frac{v}{\tau_{f i}}, \\
& I_{s o}=\left(1-H\left(V_{m}-\theta_{w}\right)\right) \frac{V_{m}-V_{m}^{o}}{\tau_{o}}+\frac{H\left(V_{m}-\theta_{w}\right)}{\tau_{s o}}, \\
& I_{s i}=-H\left(V_{m}-\theta_{w}\right) \frac{w s}{\tau_{s i}},
\end{aligned}
$$

with $H$ the standard Heaviside function. The three gate variables appearing in these equations are themselves solutions of

$$
\begin{aligned}
\partial_{t} v & =\left(1-H\left(V_{m}-\theta_{v}\right)\right) \frac{v_{\infty}-v}{\tau_{v}^{-}}-H\left(V_{m}-\theta_{v}\right) \frac{v}{\tau_{v}^{+}}, \\
\partial_{t} w & =\left(1-H\left(V_{m}-\theta_{w}\right)\right) \frac{w_{\infty}-w}{\tau_{w}^{-}}-H\left(V_{m}-\theta_{w}\right) \frac{w}{\tau_{w}^{+}}, \\
\partial_{t} s & =\frac{1+\tanh \left(k_{s}\left(V_{m}-V_{m}^{s}\right)\right)-2 s}{2 \tau_{s}} .
\end{aligned}
$$

We consider the same parameter values as in [9], except for that determining the duration of the plateau. Indeed, we change the values of the parameter $\tau_{s o}$ in order to reduce the action potential duration for epicardial, endocardial and mid-myocardial cells selectively. This heterogeneity is considered in the left ventricle, for the positivity of the T-wave in the electrocardiogram. In the right ventricle, cells are considered as homogeneous and their parameters are taken as in the left ventricle epicardium. We refer to [66] for more detail.

External stimulus $I^{a p p}$ The cells need an activation to become depolarized. Physiologically, the activation wave starts at the sinus node in the right atrium and propagates to the ventricles through the atrioventricular node before reaching the bundle of His, which activates the septum and the Purkinje network to stimulate all the endocardium. Our model does not include the atria - see [66] for a full coupling - and in order to model the electrical activation wave in an appropriate and simple way, we stimulate a part of the endocardium. We apply a given volume current density to a thin sub-endocardial layer of the ventricles during a small period of time $t_{\text {stim }}$. Then, for each simulation the applied current $I^{a p p}$ - which is summed to the ionic current $I_{\text {tot }}^{i o n}$ in System (71) - is a space-time dependent function of the form

$$
I^{a p p}(\underline{\xi}, t)=\left\{\begin{array}{cc}
I_{0}^{a p p}(\underline{\xi}, t) & \forall \underline{\xi} \in \Omega_{\text {stim }}, \forall t \in\left[0, t_{\text {stim }}\right], \\
0 & \text { otherwise. }
\end{array}\right.
$$

where $\Omega_{\text {stim }} \subset \Omega_{0}$ corresponds to the stimulation area determined using the strategy proposed in [6]. This consists in modeling the Purkinje fibers by using a predefined stimulus pattern, 

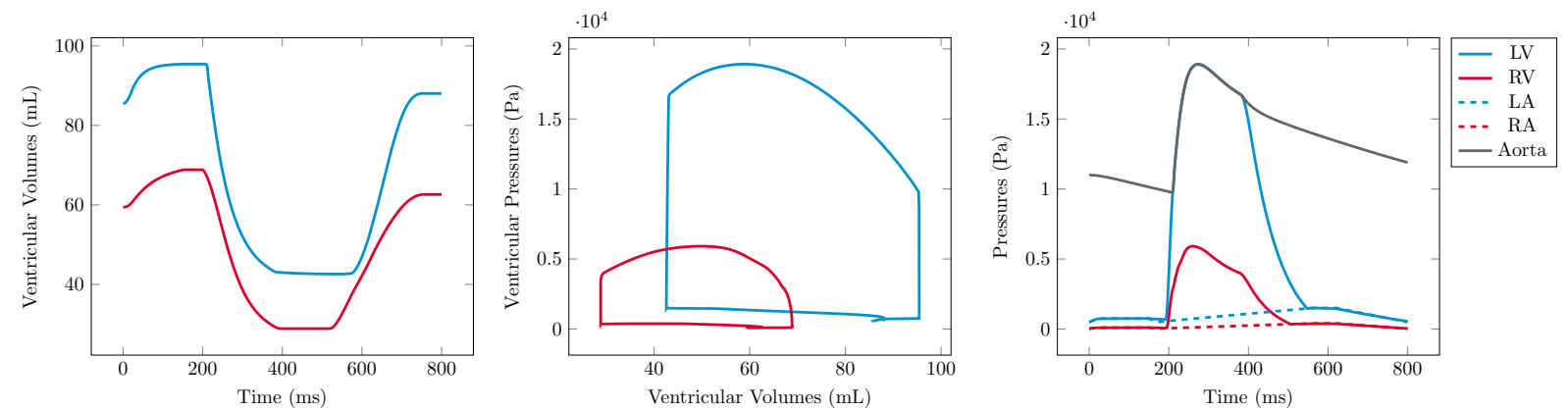

Figure 5: Volumes of cavities (left), pressure-volume loops (middle), and pressures in both cavities (right).

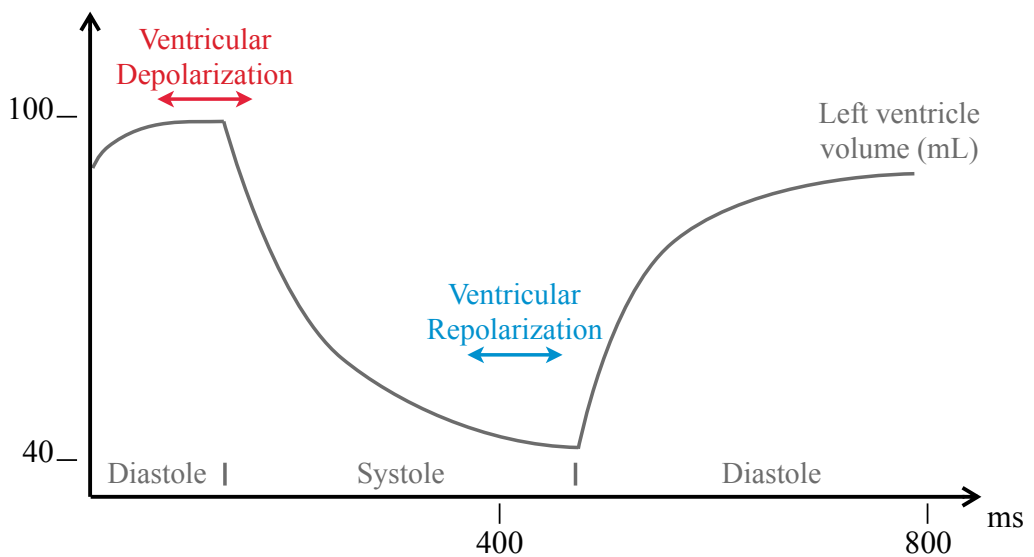

Figure 6: Left ventricular volume during a cardiac cycle with typical timings of ventricular depolarization and repolarization.

where a time-dependent thin subendocardial layer is activated using the external current for $5 \mathrm{~ms}$ in both ventricles, see Figure 8 at time $195 \mathrm{~ms}$.

Numerical methods The space and time discretizations used in the simulations correspond to the approach proposed in [6]. All the numerical procedures have been implemented in the finite element library named FELiScE ${ }^{2}$ developed at Inria. Space discretization is performed by applying the finite element method with the bidomain model equations (71). The resulting system is discretized in time by combining a second-order implicit scheme - backward differentiation formulae, see [59] - with an explicit treatment of the ionic currents.

\subsubsection{Coupling strategy}

The mechanical displacements are required for the simulation of (71). To obtain them, we consider the biomechanical model presented in [13] where the electrical stimulation is considered as an input, with numerical simulations performed using the heartLab code developed at Inria, see also [11] for more detail on the mechanical model and the coupling with an approximate circulation model. A coupled solution was obtained by a fixed point procedure with iterations between the biomechanical model and the bidomain model. In order to initialize the iterations,

\footnotetext{
${ }^{2}$ http://felisce.gforge.inria.fr
} 


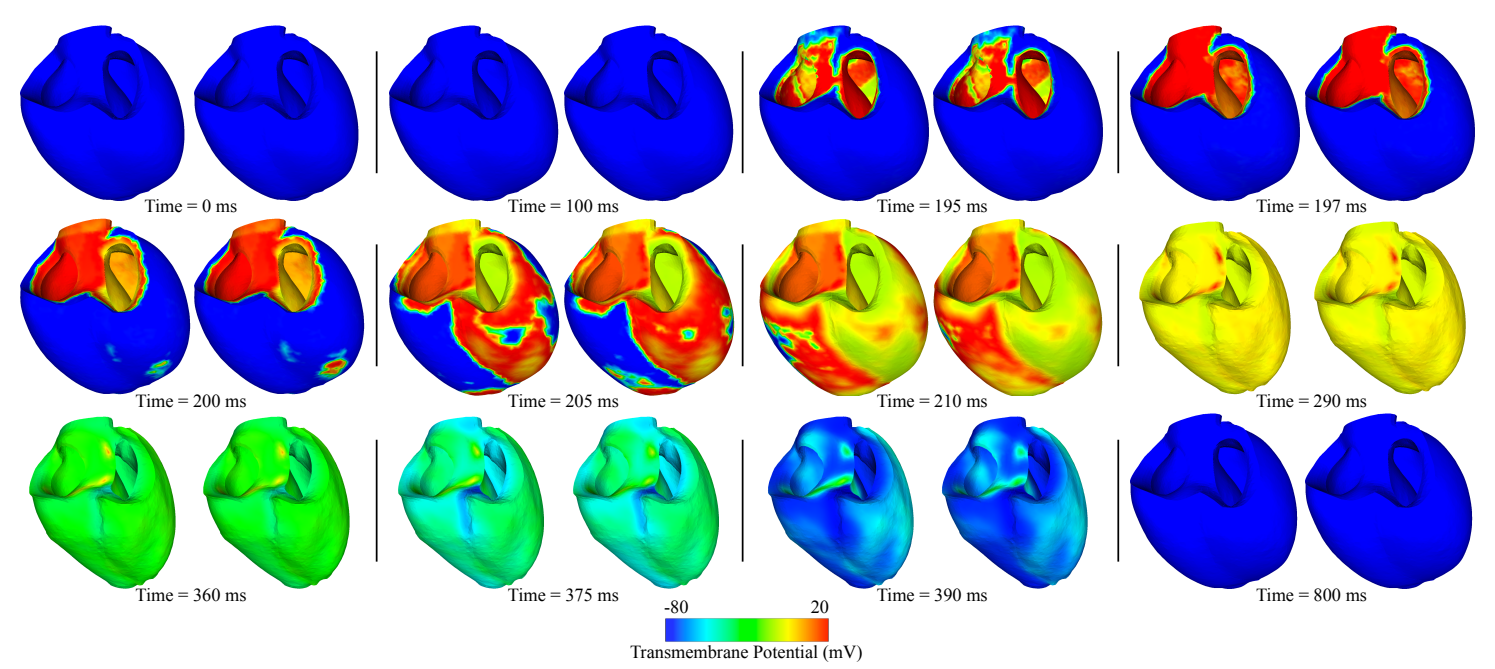

Figure 7: Comparison between the results of the bidomain model without (left) and with (right) deformations.

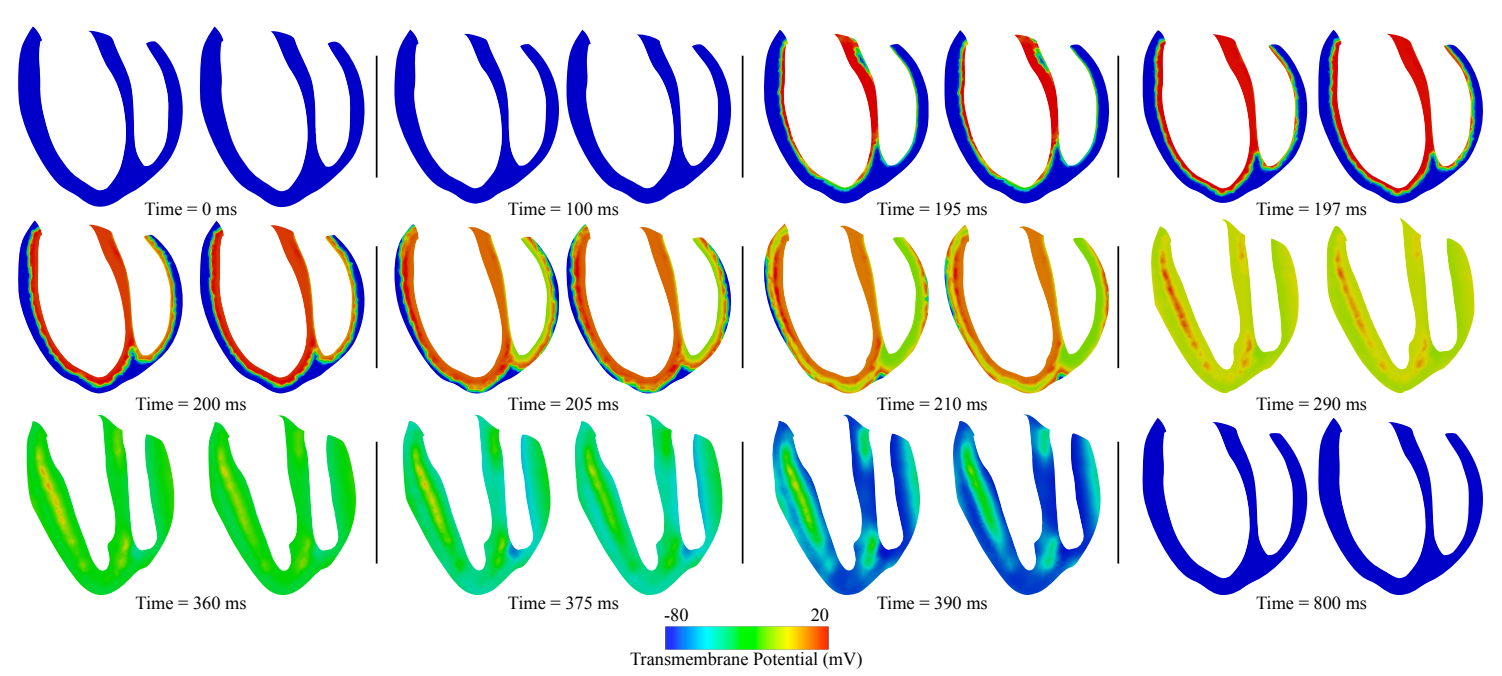

Figure 8: Comparison between the results of the bidomain model without (left) and with (right) deformations in a cut of the heart. 
we use the results of the bidomain model $(64)$ when $\underline{\underline{F}}(\underline{\xi}, t)=\underline{\underline{I}}$. Figure 5 displays the evolution of various indicators for both ventricles during one cardiac cycle obtained with the last biomechanical simulation. All these indicators are in good agreement with physiological values.

\subsubsection{Simulation results and discussion}

To facilitate the discussion, Figure 6 illustrates the relationship between the main stages in cardiac electrophysiology and mechanics by showing a typical evolution of the left ventricular volume during a cardiac cycle with the timings of ventricular depolarization and repolarization. Depolarization occurs at end-diastole, which corresponds to the stage of the cardiac cycle when the ventricle chambers are largest - hence, so are the mechanical deformations. By contrast, repolarization occurs at end-systole when the chambers are smallest.

We display the simulation results in Figure 7 and in Figure 8 for a cut of the heart, where we compare for each time considered the potential maps obtained with and without taking the deformations into account, albeit applying the same deformation in the visualization to ease the comparison. The simulation starts when the atria contract and eject the blood in the ventricles, see the left diastole part in Figure 6 between $t=0$ and $190 \mathrm{~ms}$, i.e. when the volumes of the ventricles are near their maximum. The applied current $I^{a p p}$ stimulates the endocardium at $t=190 \mathrm{~ms}$. As we can see in Fig. 7 and 8 , the depolarization is faster when deformations are considered, which is explained by the fact that the signal mainly propagates through the ventricular wall thickness from the endocardium to the epicardium. Indeed, the wall thickness is thinner when the walls are stretched. The depolarizations are complete at $211 \mathrm{~ms}$ vs. $214 \mathrm{~ms}$ with and without deformations, respectively, which gives a delay of $3 \mathrm{~ms}$ on a depolarization duration of $21 \mathrm{~ms}$, i.e. a difference of $15 \%$. Near the end of depolarization (around $t=210 \mathrm{~ms}$ ), ejection begins. Before the end of ejection (around $t=365 \mathrm{~ms}$ ), repolarization occurs. We can see $(t=$ $390 \mathrm{~ms}$ ) that the repolarization appears somewhat faster when the deformations are considered, but the advance of $3 \mathrm{~ms}$ due to depolarization has to be considered. The repolarizations of both simulations are complete at $410 \mathrm{~ms}$ vs. $411 \mathrm{~ms}$ with and without deformations, respectively, which gives a small difference in the end.

We can summarize our observations by saying that the effects of deformations as represented by our model are predominantly seen in the depolarization phase, where they speed up the propagation through the wall, with an impact on the depolarization timings that is comparable - in relative value - to the deformation amplitude. We point out that this is also comparable to the impact obtained when only considering changes in the constitutive laws to account for the mechano-electrical feedback. Note that changing the geometry on which the fixed-domain electrophysiology problem is solved to one closer to end-diastolic configuration would most probably reduce the discrepancy in the depolarization phase with the solution of the model incorporating mechanical deformations. Nevertheless, this would not be satisfactory in our context where only the mechanical reference configuration (close to end-systole) is assumed to be given, and moreover in this case it would instead introduce discrepancies in the repolarization phase. In other words, if conductivity parameters are calibrated in one given configuration (end-systolic or end-diastolic), using the same parameters in a phase where the deformed geometry is far from this configuration will introduce discrepancies between the solutions of the electrophysiology problem with and without deformations.

\subsection{Electrocardiogram simulations}

An electrocardiogram (ECG) is a recording of the electrical activity of the heart $[40,76]$ measured at the outer surface of the body. This procedure - which is non-invasive and inexpensive - is the most standard diagnosis tool for cardiologists to detect cardiac pathologies. The 


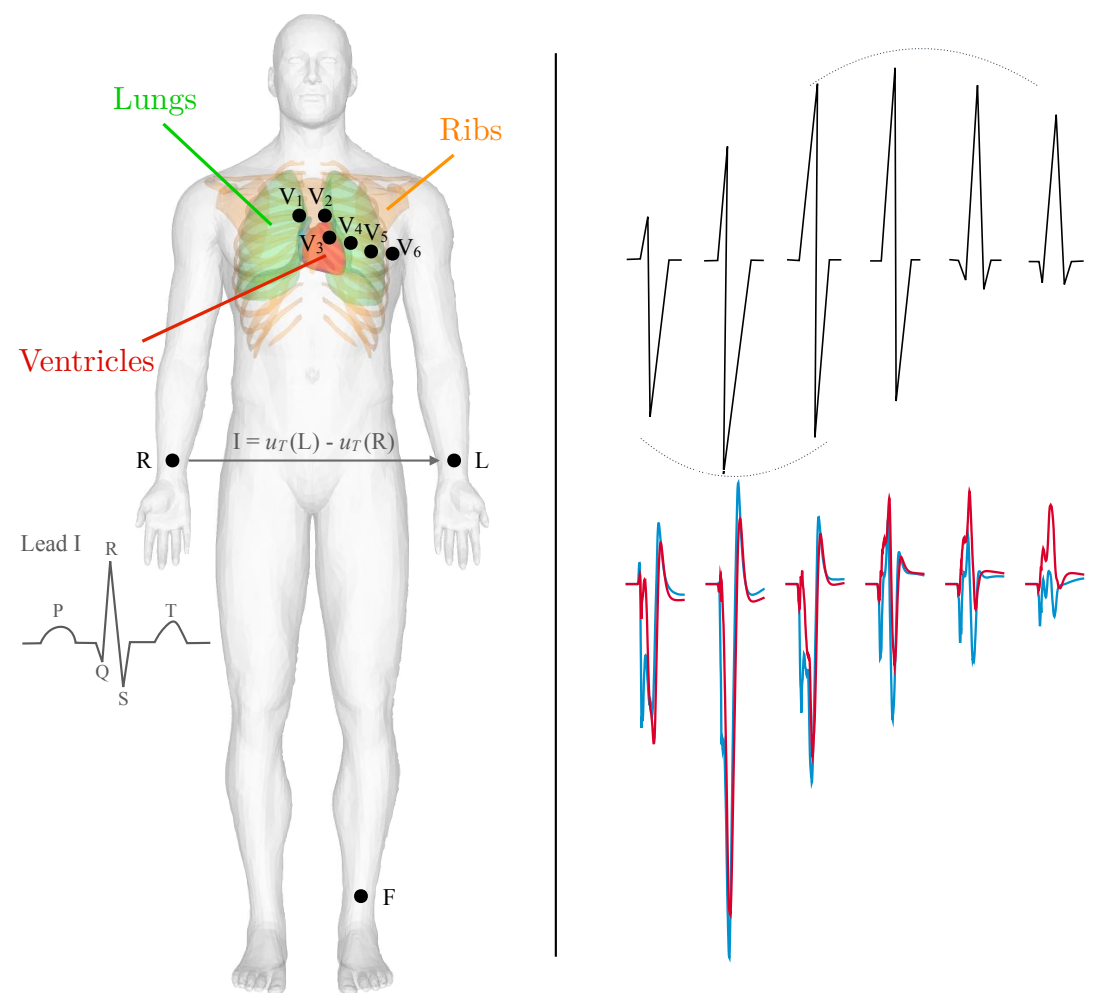

Figure 9: Left: Mesh of the torso domain $\Omega_{T}$ with the locations of the 9 electrodes. Right: $\mathrm{R}$-wave progression in the precordial leads $\mathrm{V}_{1}, \cdots, \mathrm{V}_{6}$ : schematic view from [76] (top), and simulated ECGs (bottom). Bidomain model simulations with (blue) and without (red) deformations.

\begin{tabular}{ccc}
\hline \hline$\sigma_{T}^{\text {body }}$ & $\sigma_{T}^{\text {bones }}$ & $\sigma_{T}^{\text {lungs }}$ \\
\hline \hline $3.010^{-4}$ & $1.210^{-4}$ & $2.010^{-5}$ \\
\hline
\end{tabular}

Table 2: Torso conductivity parameters (all in S.cm ${ }^{-1}$ ).

literature on the simulation of ECG is abundant, see e.g. $[5,30,35,38]$ and references therein. The simulation of 12-lead ECGs based on partial differential equations (PDEs) - as proposed here - appeared during the last decade $[6,41,53,55,72]$, more recently than simulations based on cellular automata [77].

\subsubsection{Coupling with the body}

To model an ECG, the coupling between the heart and the rest of the body must be taken into account. We consider a diffusion problem in the rest of the body, see Figure 9-left for the body domain, i.e.

$$
-\operatorname{div}\left(\sigma_{T} \underline{\nabla} u_{T}\right)=0, \quad \text { in } \Omega_{T}
$$

where the electrical conductivity coefficient $\sigma_{T}$ takes different values in the ribs and the lungs, see [10] and Table 2. On the body surface $\partial \Omega_{T}^{e x t}$ - corresponding to the skin - a homogeneous Neumann boundary condition is imposed

$$
\sigma_{T} \underline{\nabla} u_{T} \cdot \underline{n}=0, \quad \text { on } \partial \Omega_{T}^{e x t}
$$

i.e., we assume that the body is electrically isolated. 
Last but not least, the transmission conditions at the heart-body interface $\partial \Omega_{H}$ must be adequately chosen. In this work, we consider that the extracellular current does not flow through the pericardium, i.e. , the heart is considered as isolated,

$$
\left(\underline{\underline{\sigma}}^{(e)} \cdot \underline{\nabla} u^{(e)}\right) \cdot \underline{n}=0, \quad \text { on } \partial \Omega_{H}
$$

and that we have a perfect electric contact between the heart and the torso domains

$$
u_{T}=u_{e}, \quad \text { on } \Omega_{H} .
$$

Condition (73) is known to have a limited impact on the shape of the ECG - see e.g. [6] - and allows to solve the full system as a one-way coupled problem, which dramatically reduces its computational cost.

An additional stage is necessary to obtain simulated ECGs with the bidomain model when deformations are considered, due to the deformation of the heart domain in the coupling, as the thorax domain is assumed to be fixed. We handle this new difficulty by projecting the solution obtained with the deformations onto $\partial \Omega_{H}$, the boundary of the thorax domain adjacent to the heart.

\subsubsection{Electrocardiogram computation}

A standard 12-lead ECG is obtained with 9 electrodes located on the body surface, see Figure 9-left,

$$
\begin{aligned}
\mathrm{I} & =u_{T}(L)-u_{T}(R), & \mathrm{aVR} & =1.5\left(u_{T}(R)-u_{w}\right), \\
\mathrm{II} & =u_{T}(F)-u_{T}(R), & \mathrm{aVL} & =1.5\left(u_{T}(L)-u_{w}\right), \\
\mathrm{III} & =u_{T}(F)-u_{T}(L), & \mathrm{aVF} & =1.5\left(u_{T}(F)-u_{w}\right), \\
\mathrm{V}_{1} & =u_{T}\left(\mathrm{~V}_{1}\right)-u_{w}, & \mathrm{~V}_{4} & =u_{T}\left(\mathrm{~V}_{4}\right)-u_{w}, \\
\mathrm{~V}_{2} & =u_{T}\left(\mathrm{~V}_{2}\right)-u_{w}, & \mathrm{~V}_{5} & =u_{T}\left(\mathrm{~V}_{5}\right)-u_{w}, \\
\mathrm{~V}_{3} & =u_{T}\left(\mathrm{~V}_{3}\right)-u_{w}, & \mathrm{~V}_{6} & =u_{T}\left(\mathrm{~V}_{6}\right)-u_{w},
\end{aligned}
$$

where $u_{w}=\frac{1}{3}\left(u_{T}(L)+u_{T}(R)+u_{T}(F)\right)$ is the Wilson potential [40]. The leads I, II and III are called the limb leads, aVR, aVL and aVF the augmented limb leads. The leads $\mathrm{V}_{i}$, for $i \in\{1, \cdots, 6\}$ are called the precordial leads. A lead signal is decomposed into 5 waves, see Figure 9-left for an example with the lead I:

- the P-wave which represents the atrial depolarization,

- the QRS complex which represents the ventricular depolarization,

- the QT segment which corresponds to the plateau of ventricular action potential,

- the T-wave which represents the ventricular repolarization.

As we do not consider the atria in this work, we do not have the $\mathrm{P}$ wave, see [66] for complete simulated ECGs.

The simulated ECGs can be validated against numerous criteria used to assess real electrocardiograms. Those considered in this work are summarized in Table 3, see [66] for a more complete survey. 


\begin{tabular}{cccc}
\hline \hline $\begin{array}{c}\text { Wave/ } \\
\text { Interval }\end{array}$ & Description & $\begin{array}{c}\text { Without } \\
\text { displacements }\end{array}$ & $\begin{array}{c}\text { With } \\
\text { displacements }\end{array}$ \\
\hline \hline \multirow{3}{*}{ R-wave } & limb leads $\leq 2 \mathrm{mV}$ & $\checkmark$ & $\checkmark(\approx \mathrm{III})$ \\
& precordial leads $\leq 3 \mathrm{mV}$ & $\checkmark$ & $\checkmark$ \\
& always $\geq 0, \leq 0$ in aVR & $\checkmark$ & $\checkmark$ \\
& R-wave progression, see Fig. 9-right & $\checkmark$ & $\checkmark\left(\approx \mathrm{V}_{6}\right)$ \\
\hline T-wave & $\geq 0$ I, II, V3 to V6 & $\checkmark(\approx \mathrm{II})$ & $\checkmark$ \\
& $\leq 0$ aVR (follow the QRS) & $\checkmark$ & $\checkmark$ \\
\hline
\end{tabular}

Table 3: Criteria for typical ECGs [76], compared with simulated ECGs of Fig. 10.
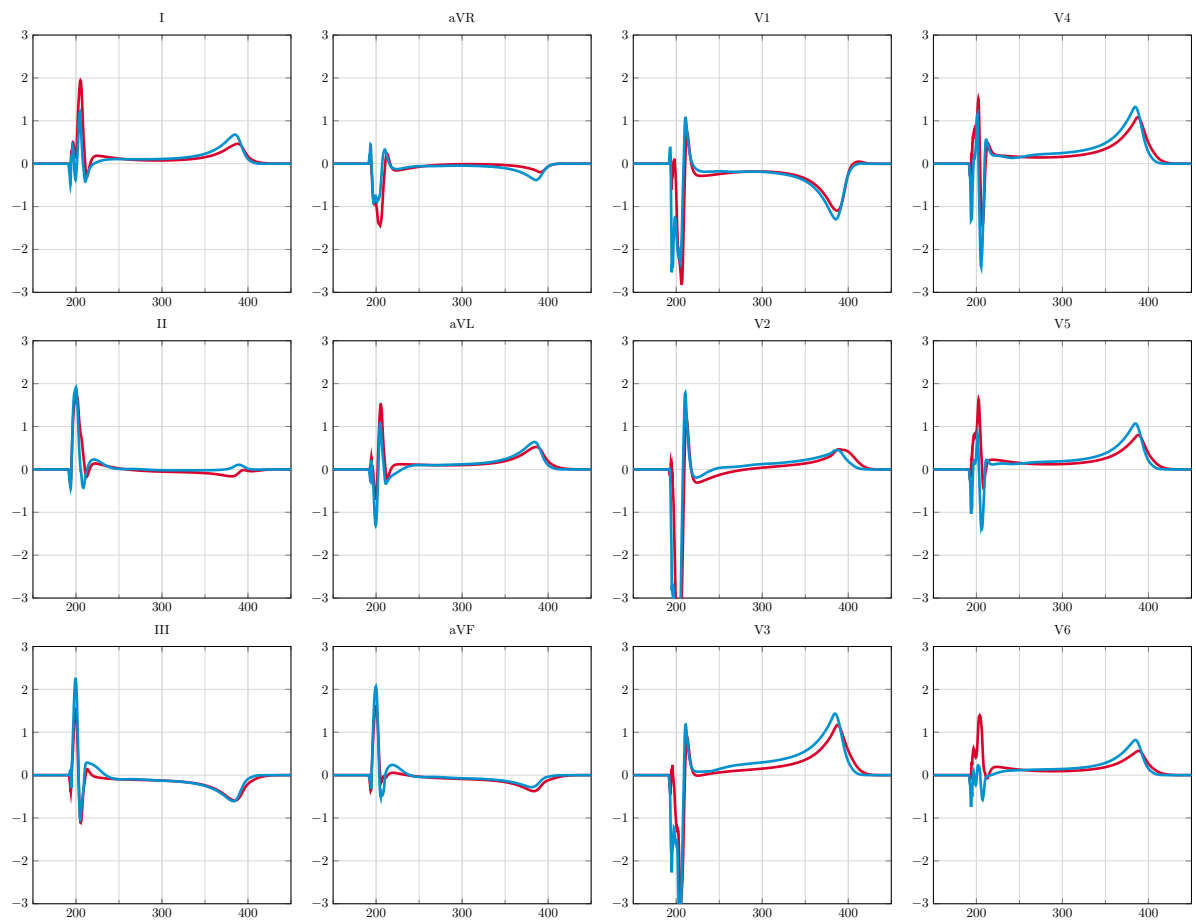

Figure 10: Simulated ECGs with (blue) and without (red) deformations. 

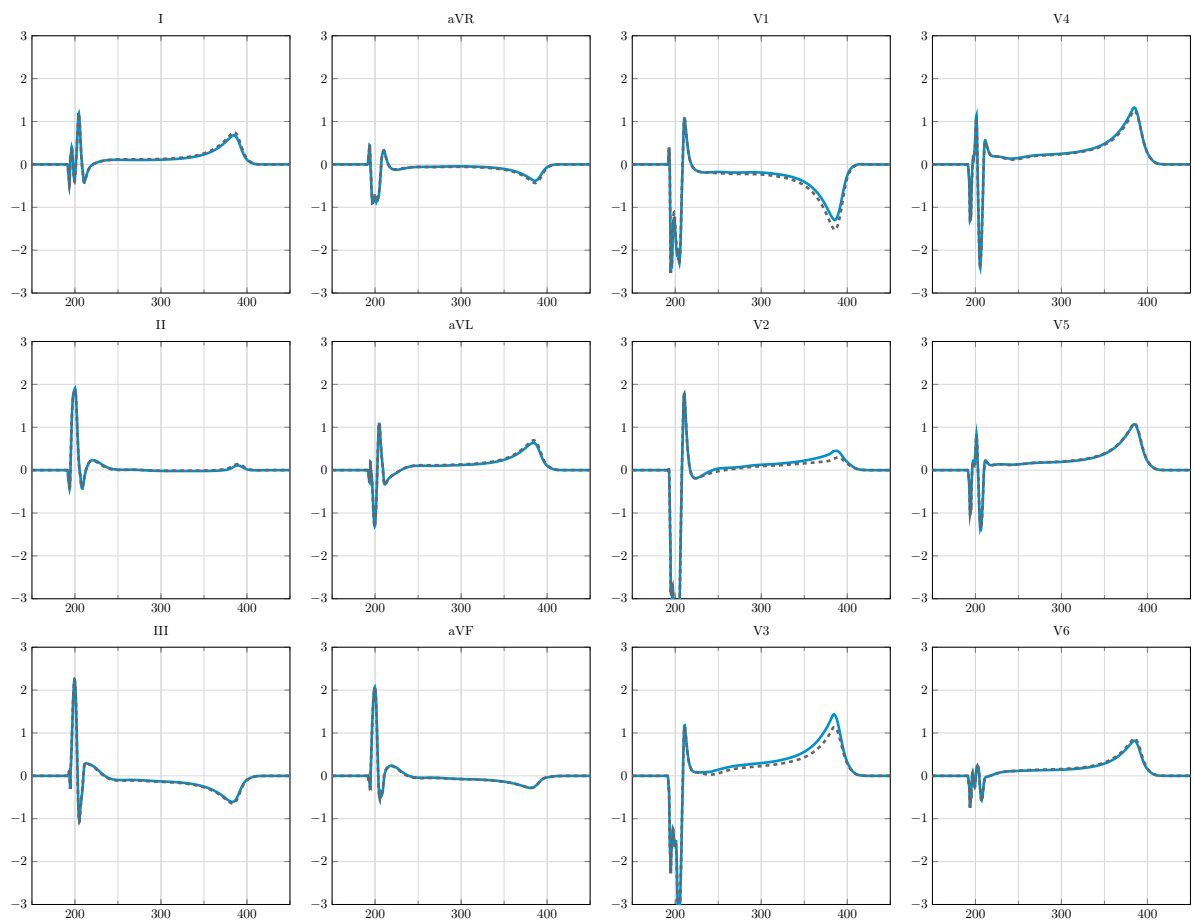

Figure 11: Simulated ECGs with deformations, and with (blue) and without (dashed gray) projection on the thorax.

\subsubsection{Results}

Figure 10 displays the simulated ECGs with (blue) and without (red) deformations. Table 3 shows that these simulated ECGs are quite realistic. As already discussed in [66], in the simulated ECGs the QRS complex is shorter than physiologically expected. Numerous factors including the geometry, the heart position inside the torso, the orientation of muscle fibers, the choice of ionic model and the model parameters (for instance heart and body conductivities) make the simulation of realistic electrocardiograms very difficult, see $[56,57,60]$. As our primary objective in this work is to assess the effects of mechanical deformations on the bidomain model - for which simulated ECGs are only one specific indicator - this point is not a limitation in itself, as it impacts both simulations (with or without mechanical effects). Concerning the case with deformation, some oscillations are observed and the precordial lead $\mathrm{V}_{6}$ is not perfect. This could be improved by a more adapted applied current $I^{a p p}$ and a better choice of the many parameters - which were adjusted to the case without deformation - but it is not the objective in this article. The main difference between the two simulations is the duration of the $\mathrm{R}$-wave in the limb leads, which is smaller when the displacements are considered. This is due to the faster depolarization as explained in Section 4.2.2.

Next, we want to study the impact of the projecting step onto the thorax contact surface. Figure 11 gives the ECGs with (blue line, same as in Figure 10) and without (dashed gray line) projection. The difference on the depolarization (QRS complex) is very small. This can be explained by the fact that during the depolarization the heart inflates along the normal direction of the epicardium. This implies that the impact of the projection can be neglected. The impact on the repolarization (T-wave) is more pronounced on the first three precordial leads. This can be explained by the fact that the repolarization occurs when the heart chambers are smaller and twisted under the action of the cardiac torsion. It does not seem necessary to do this projecting 
step - which is computationally expensive - in view of these results.

Finally, our simulations reveal a significant - although not predominant - impact of mechanical deformations on the ECGs. One natural perspective would be to compare our mechanically deformed bidomain model - which takes into account the heart deformation in conservation laws - with the classical mechano-electrical feedback model in a realistic case of ECG simulation.

\section{Conclusions}

We have followed a formal homogenization approach to investigate the effects of mechanical deformations on electrophysiology, and more specifically for electrophysiology models relying on a bidomain description of ionic motion at the microscopic level. To that purpose we have extended these microscopic equations to take into account the mechanical deformations, and proceeded by recasting the problem in the framework of classical two-scale homogenization in periodic media and identifying the equations satisfied by the first coefficients in the formal expansions. In so doing, we have not taken for granted the usual - albeit not easily justified - assumptions classically made in the derivation of the macroscopic bidomain equations - i.e. negligible diffusion compared to drift in ionic equations, and constant effective conductivities - and this results in homogenized equations that describe ionic concentrations and fluxes species by species, in addition to electric potentials. Nevertheless, the standard approximations can be considered in a second stage to derive equations on potentials only. In all cases, the homogenized equations reveal some interesting effects related to the microstructure - and associated with a specific cell problem to be solved to obtain the macroscopic conductivity tensors - in which mechanical deformations play a non-trivial role, i.e. do not simply lead to a standard bidomain problem posed in the deformed configuration.

We have then presented detailed numerical illustrations of the resulting homogenized model with coupled cardiac electrical-mechanical simulations, albeit without taking into account the abundantly-investigated effect of mechanical deformations in ionic models, in order to focus here on other effects. And in fact our numerical results indicate - both in the $3 \mathrm{D}$ electrical potentials and in the associated ECGs - that these other effects are numerically of a comparable order, and therefore cannot be disregarded.

Relevant perspectives and extensions of this work include further investigations to justify the approximation made in (70) to avoid solving a deformation-dependent cell problem at every point in the domain to compute the conductivity tensor, for which we conjecture that an asymptotic analysis, either with respect to the aspect ratio of the cells, or to the GreenLagrange deformation tensor $\frac{1}{2}\left(\underline{F}^{T} \cdot \underline{F}-\underline{\underline{I}}\right)$ (i.e. a local measure of departure from rigid body deformation), may provide some interesting insight. From a modeling standpoint, a more refined - and realistic - homogenization problem could take into account gap junctions that hinder ionic fluxes between intracellular regions of adjacent cells [26], which would break the symmetry between intra- and extracellular equations.

\section{References}

[1] Ismail Adeniran, Jules C. Hancox, Henggui Zhang, et al. Effect of cardiac ventricular mechanical contraction on the characteristics of the ECG: A simulation study. Journal of Biomedical Science and Engineering, 6(12):47, 2013.

[2] R. Aliev and A. Panfilov. A simple two-variable model of cardiac excitation. Chaos, Solitons \& Fractals, 3(7):293-301, 1996. 
[3] Grégoire Allaire. Shape optimization by the homogenization method, volume 146. Springer Science \& Business Media, 2012.

[4] Sergio Alonso, Markus Bär, and Blas Echebarria. Nonlinear physics of electrical wave propagation in the heart: a review. Reports on Progress in Physics, 79(9):096601, 2016.

[5] M. Bishop and G. Plank. Bidomain ecg simulations using an augmented monodomain model for the cardiac source. Biomedical Engineering, IEEE Transactions on, 58(8):22972307, 2011.

[6] M. Boulakia, S. Cazeau, M.A. Fernández, J.-F. Gerbeau, and N. Zemzemi. Mathematical modeling of electrocardiograms: a numerical study. Annals of Biomedical Engineering, 38(3):1071-1097, 2010.

[7] M. Boulakia, M.A. Fernández, J-F. Gerbeau, and N. Zemzemi. A coupled system of PDEs and ODEs arising in electrocardiograms modeling. Applied Mathematics Research eXpress, 2008.

[8] M. Briane. Three models of non periodic fibrous materials obtained by homogenization. RAIRO-Modélisation mathématique et analyse numérique, 27(6):759-775, 1993.

[9] A. Bueno-Orovio, E.M. Cherry, and F.H. Fenton. Minimal model for human ventricular action potentials in tissue. Journal of Theretical Biology, 253:544-560, 2008.

[10] M.L. Buist and A.J. Pullan. The effect of torso impedance on epicardial and body surface potentials: A modeling study. IEEE Transactions on Biomedical Engineering, 50(7):816 $824,2003$.

[11] R. Chabiniok, P. Moireau, P.F. Lesault, A. Rahmouni, J.F. Deux, and D. Chapelle. Estimation of tissue contractility from cardiac cine-MRI using a biomechanical heart model. Biomechanics and Modeling in Mechanobiology, 11(5):609-630, 2011.

[12] R. Chabiniok, V.Y. Wang, M. Hadjicharalambous, L. Asner, J. Lee, M. Sermesant, E. Kuhl, A.A. Young, P. Moireau, M.P. Nash, D. Chapelle, and D.A. Nordsletten. Multiphysics and multiscale modelling, data-model fusion and integration of organ physiology in the clinic: ventricular cardiac mechanics. Interface Focus, 6(2), 2016.

[13] D. Chapelle, P. Le Tallec, P. Moireau, and M. Sorine. Energy-preserving muscle tissue model: formulation and compatible discretizations. International Journal for Multiscale Computational Engineering, 10(2):189-211, 2012.

[14] P. Colli Franzone, L. F. Pavarino, and S. Scacchi. Effects of mechanical feedback on the stability of cardiac scroll waves: A bidomain electro-mechanical simulation study. Chaos: An Interdisciplinary Journal of Nonlinear Science, 27(9):1-15, 2017.

[15] P. Colli Franzone, L F. Pavarino, and S. Scacchi. A numerical study of scalable cardiac electro-mechanical solvers on hpc architectures. Frontiers in Physiology, 9:1-16, 2018.

[16] P. Colli Franzone, L.F. Pavarino, and S. Scacchi. Bioelectrical effects of mechanical feedbacks in a strongly coupled cardiac electro-mechanical model. Mathematical Models and Methods in Applied Sciences, 26(01):27-57, 2016.

[17] P. Colli Franzone, L.F. Pavarino, and B. Taccardi. Simulating patterns of excitation, repolarization and action potential duration with cardiac Bidomain and Monodomain models. Mathematical Biosciences, 197(1):35-66, 2005. 
[18] P. Colli Franzone and G. Savaré. Degenerate evolution systems modeling the cardiac electric field at micro and macroscopic level. Progress in Nonlinear Differential Equations and Their Applications, 50:49-78, 2002.

[19] A. Collin and S. Imperiale. Mathematical analysis and 2-scale convergence of a heterogeneous microscopic bidomain model. Mathematical Models and Methods in Applied Sciences, 28(05):979-1035, 2018.

[20] M. Courtemanche, R.J. Ramirez, and S. Nattel. Ionic mechanisms underlying human atrial action potential properties: insights from a mathematical model. American Journal of Physiology, 275:301-321, 1998.

[21] M. Favino, S. Pozzi, S. Pezzuto, F.W. Prinzen, A. Auricchio, and R. Krause. Impact of mechanical deformation on pseudo-ECG: a simulation study. EP Europace, 18(4):77-84, 2016.

[22] R. Fitzhugh. Impulses and physiological states in theoretical models of nerve membrane. Biophysical Journal, 1(6):445-466, 1961.

[23] P. Frey. Yams: A fully automatic adaptive isotropic surface remeshing procedure. Technical report 0252, Inria, Rocquencourt, France, Nov. 2001.

[24] PL George. Tetmesh-ghs3d, tetrahedral mesh generator. INRIA User's Manual, INRIA (Institut National de Recherche en Informatique et Automatique), France, 83, 2004.

[25] Christophe Geuzaine and Jean-François Remacle. Gmsh: A 3-d finite element mesh generator with built-in pre-and post-processing facilities. International journal for numerical methods in engineering, 79(11):1309-1331, 2009.

[26] P.E. Hand and C.S. Peskin. Homogenization of an electrophysiological model for a strand of cardiac myocytes with gap-junctional and electric-field coupling. Bulletin of Mathematical Biology, 72:1408-1424, 2010.

[27] Sarah N Healy and Andrew D McCulloch. An ionic model of stretch-activated and stretchmodulated currents in rabbit ventricular myocytes. Europace, 7(s2):S128-S134, 2005.

[28] F. Hecht. New development in FreeFem++. Journal of Numerical Mathematics, 20(34):251-265, 2012.

[29] A.L. Hodgkin and A.F. Huxley. A quantitative description of membrane current and its application to conduction and excitation in nerve. Journal of Physiology, 117(4):500-544, 1952.

[30] G. Huiskamp. Simulation of depolarization in a membrane-equations-based model of the anisotropic ventricle. Biomedical Engineering, IEEE Transactions on, 45(7):847-855, 1998.

[31] M.-P. Jolly. Automatic segmentation of the left ventricle in cardiac MR and CT images. International Journal of Computer Vision, 70(2):151-163, 2006.

[32] J. Keener. An eikonal-curvature equation for action potential propagation in myocardium. Journal of Mathematical Biology, 29:629-651, 1991.

[33] J. Keener and J. Sneyd. Mathematical Physiology. Springer, 2004. 
[34] R.H. Keldermann, M.P. Nash, and A.V. Panfilov. Modeling cardiac mechano-electrical feedback using reaction-diffusion-mechanics systems. Physica D, 238:1000-1007, 2008.

[35] D. Keller, G. Seemann, D. Weiss, D. Farina, J. Zehelein, and O. Dössel. Computer based modeling of the congenital long-QT 2 syndrome in the visible man torso: From genes to ECG. In Engineering in Medicine and Biology Society, 200\%. EMBS 2007. 29th Annual International Conference of the IEEE, pages 1410-1413, 2007.

[36] B.J. Kirby. Micro- and Nanoscale Fluid Mechanics: Transport in Microfluidic Devices. Cambridge University Press, 2010.

[37] E. Konukoglu, O. Clatz, B. H. Menze, B. Stieltjes, M.-A. Weber, E. Mandonnet, H. Delingette, and N. Ayache. Image guided personalization of reaction-diffusion type tumor growth models using modified anisotropic eikonal equations. Medical Imaging, IEEE Transactions, 29(1):77-95, 2010.

[38] G.T. Lines, M.L. Buist, P. Grottum, A.J. Pullan, J. Sundnes, and A. Tveito. Mathematical models and numerical methods for the forward problem in cardiac electrophysiology. Comput. Visual. Sci., 5(4):215-239, 2003.

[39] M. Lynch, O. Ghita, and P.F. Whelan. Automatic segmentation of the left ventricle cavity and myocardium in MRI data. Computers in biology and medicine, 36(4):389-407, 2006.

[40] J. Malmivuo and R. Plonsey. Bioelectromagnetism - Principles and Applications of Bioelectric and Biomagnetic Fields. Oxford University Press, 1995.

[41] V. Martin, A. Drochon, O. Fokapu, and J-F. Gerbeau. Magnetohemodynamics in the aorta and electrocardiograms. Physics in Medicine and Biology, 57:3177-3195, 2012.

[42] C.C. Mitchell and D.G. Schaeffer. A two-current model for the dynamics of cardiac membrane. Bulletin Math. Bio., 65:767-793, 2003.

[43] Y. Mori. From three-dimensional electrophysiology to the cable model: an asymptotic study. arXiv preprint arXiv:0901.3914, 2009.

[44] Y. Mori, J.W. Jerome, and C.S. Peskin. A three-dimensional model of cellular electrical activity. Bulletin of the Institute of Mathematics, Academia Sinica, 2(2):367-390, 2007.

[45] A. Nagler, C. Bertoglio, C.T. Stoeck, S. Kozerke, and W.A. Wall. Cardiac fibers estimation from arbitrarily spaced diffusion weighted MRI. In Functional Imaging and Modeling of the Heart, pages 198-206. Springer, 2015.

[46] J.S. Nagumo, S. Arimoto, and S. Yoshizawa. An active pulse transmission line stimulating nerve axon. Proceedings of the IEEE, 50:2061-2071, 1962.

[47] M.P. Nash and P.J. Hunter. Computational mechanics of the heart, from tissue structure to ventricular function. Journal of elasticity and the physical science of solids, 61:113-141, 2000 .

[48] M.P. Nash and A.V. Panfilov. Electromechanical model of excitable tissue to study reentrant cardiac arrhythmias. Progress in Biophysics and Molecular Biology, 85(2-3):501-522, 2004.

[49] J.C. Neu and W. Krassowska. Homogenization of syncytial tissues. Critical Reviews in Biomedical Engineering, 21(2):137-199, 1993. 
[50] A. Nygren, C. Fiset, L. Firek, J.W. Clark, D.S. Lindblad, R.B. Clark, and W.R. Giles. Mathematical model of an adult human atrial cell the role of $\mathrm{K}+$ currents in repolarization. Circulation Research, 82(1):63-81, 1998.

[51] T. O'Hara, L. Virág, A. Varró, and Y. Rudy. Simulation of the undiseased human cardiac ventricular action potential: Model formulation and experimental validation. PLoS Comput Biol, 7(5):e1002061, 2011.

[52] Alexander V Panfilov, RH Keldermann, and MP Nash. Self-organized pacemakers in a coupled reaction-diffusion-mechanics system. Physical review letters, 95(25):258104, 2005.

[53] M. Potse, B. Dubé, and R.M. Gulrajani. ECG simulations with realistic human membrane, heart, and torso models. In Engineering in Medicine and Biology Society, 2003. Proceedings of the 25th Annual International Conference of the IEEE, volume 1, pages 70-73, 2003.

[54] M. Potse, B. Dubé, J. Richer, A. Vinet, and R.M. Gulrajani. A comparison of monodomain and bidomain reaction-diffusion models for action potential propagation in the human heart. IEEE Transactions on Biomedical Engineering, 53(12):2425-2435, 2006.

[55] M. Potse, B. Dubé, and A. Vinet. Cardiac anisotropy in boundary-element models for the electrocardiogram. Medical \& biological engineering \& computing, 47(7):719-729, 2009.

[56] Mark Potse, Bruno Dubé, and Ramesh M Gulrajani. Ecg simulations with realistic human membrane, heart, and torso models. In Proceedings of the 25th Annual International Conference of the IEEE Engineering in Medicine and Biology Society (IEEE Cat. No. 03СH37439), volume 1, pages 70-73. IEEE, 2003.

[57] Mark Potse, Bruno Dubé, and Alain Vinet. Cardiac anisotropy in boundary-element models for the electrocardiogram. Medical \&f biological engineering \& computing, 47(7):719-729, 2009 .

[58] Mariya Ptashnyk. Multiscale modelling and analysis of signalling processes in tissues with non-periodic distribution of cells. Vietnam Journal of Mathematics, 45(1):295-316, 2017.

[59] A. Quarteroni, R. Sacco, and F. Saleri. Numerical mathematics, volume 37. Texts in Applied Mathematics. Springer-Verlag, Berlin, second edition., 2007.

[60] Alfio Quarteroni, Luca Formaggia, and Alessandro Veneziani. Complex systems in biomedicine. Number BOOK. Springer, 2006.

[61] Alfio Quarteroni, Toni Lassila, Simone Rossi, and Ricardo Ruiz-Baier. Integrated heartcoupling multiscale and multiphysics models for the simulation of the cardiac function. Computer Methods in Applied Mechanics and Engineering, 314:345-407, 2017. Special Issue on Biological Systems Dedicated to William S. Klug.

[62] G. Richardson. A multiscale approach to modelling electrochemical processes occurring across the cell membrane with application to transmission of action potentials. Mathematical Medicine and Biology, 26(3):201-224, 2009.

[63] G. Richardson and J. Chapman. Derivation of the bidomain equations for a beating heart with a general microstructure. SIAM Journal on Applied Mathematics, 71(3):657-675, 2011. 
[64] J.-M. Rogers and A.-D. McCulloch. A collocation-Galerkin finite element model of cardiac action potential propagation. IEEE Transactions on Biomedical Engineering, 41(8):743757, 1994.

[65] F.B. Sachse. Computational Cardiology: Modeling of Anatomy, Electrophysiology and Mechanics. Springer-Verlag, 2004.

[66] E. Schenone, A. Collin, and J.-F. Gerbeau. Numerical simulation of electrocardiograms for full cardiac cycles in healthy and pathological conditions. International Journal for Numerical Methods in Biomedical Engineering, 2015.

[67] D.F. Scollan, A. Holmes, J. Zhang, and R.L. Winslow. Reconstruction of cardiac ventricular geometry and fiber orientation using magnetic resonance imaging. Annals of biomedical engineering, 28(8):934-944, 2000.

[68] D.D. Streeter. Gross morphology and fiber geometry of the heart. Handbook Physiology, The cardiovascular system, 1:61-112, 1979.

[69] R. Temam and A. Miranville. Mathematical Modeling in Continuum Mechanics. Cambridge University Press, second edition, 2005.

[70] K.H.W.J. ten Tusscher, D. Noble, P.J. Noble, and A.V. Panfilov. A model for human ventricular tissue. American Journal of Physiology-Heart and Circulatory Physiology, 286(4):H1573-H1589, 2004.

[71] K.H.W.J. ten Tusscher and A.V. Panfilov. Alternans and spiral breakup in a human ventricular tissue model. American Journal of Physiology-Heart and Circulatory Physiology, 291(3):H1088-H1100, 2006.

[72] M.-C. Trudel, B. Dubé, M. Potse, R.M. Gulrajani, and L.J. Leon. Simulation of QRST integral maps with a membrane-based computer heart model employing parallel processing. IEEE Transactions on Biomedical Engineering, 51(8):1319-1329, 2004.

[73] L. Tung. A bi-domain model for describing ischemic myocardial d-c potentials. PhD thesis, Massachusetts Institute of Technology. Dept. of Electrical Engineering and Computer Science., 1978.

[74] F. Vadakkumpadan, H. Arevalo, C. Ceritoglu, M. Miller, and N. Trayanova. Image-based estimation of ventricular fiber orientations for personalized modeling of cardiac electrophysiology. Medical Imaging, IEEE Transactions on, 31(5):1051-1060, 2012.

[75] Samuel T Wall, Julius M Guccione, Mark B Ratcliffe, and Joakim S Sundnes. Electromechanical feedback with reduced cellular connectivity alters electrical activity in an infarct injured left ventricle: a finite element model study. American Journal of Physiology-Heart and Circulatory Physiology, 302(1):H206-H214, 2012.

[76] F. Wartak. Electrocardiogram interpretation. Medical Education Systems, 1975.

[77] D. Wei, O. Okazaki, K. Harumi, E. Harasawa, and H. Hosaka. Comparative simulation of excitation and body surface electrocardiogram with isotropic and anisotropic computer heart models. Biomedical Engineering, IEEE Transactions on, 42(4):343-357, 1995.

[78] Y. Zheng, A. Barbu, B. Georgescu, M. Scheuering, and D. Comaniciu. Fast automatic heart chamber segmentation from 3D CT data using marginal space learning and steerable features. In Computer Vision, 200\%. ICCV 200\%. IEEE 11th International Conference on, pages 1-8. IEEE, 2007. 